\title{
Random Point Processes and DLR Equations
}

\author{
Yu. M. Suhov ${ }^{\star}$ \\ Centre de Physique Théorique, CNRS Marseille, and U.E.R. de Marseille-Luminy, \\ F-13274 Marseille Cedex 2, France
}

\begin{abstract}
We prove the uniqueness of a solution of the Dobrushin-LanfordRuelle equation for random point processes when the generating function (interaction potential) has no hard cores, is non-negative and rapidely decreasing.
\end{abstract}

\section{Introduction}

One of the interesting problems in the theory of random processes (r.p.) and random fields (r.f.) which is inspired by mathematical physics (more precisely, by the theory of phase transitions in systems with infinitely many degrees of freedom) is that of their description. The first formulation and motivation of this problem was given by Dobrushin who considered the problem of finding r.f. with a given system of the conditional probabilities [1-2], proved some sufficient conditions of the existence and uniqueness of such r.f. [1-6] and gave examples of non-uniqueness [4].

An independent approach was proposed by Lanford and Ruelle [7-8]. The Lanford-Ruelle approach is more immediate from the point of view of statistical mechanics: given a potential describing the "interaction" of single values of r.f., how many r.f. correspond to it? The uniqueness of the solution of this problem is associated with the absence of the phase transitions in the infinite system with this "interaction".

On the other hand, Dobrushin's approach was developped in [9-11] where the problem of describing the system of conditional probabilities was considered. It turned out that under same general conditions a system of conditional probabilities admits a so-called Gibbs description in terms of a function (or a family of functions) interpreted as a "generalized potential" of interaction of single values of a r.f. We shall call this function the "generating function" (g.f.) of the r.f. The results of [9-11] unite both approaches mentioned above. One of the

* Permanent address: Institute of Problems of Information Transmission, USSR Academy of Sciences, 8 Build 2, Aviamotornaja av., 111024 Moscow, USSR 
advantages of the Gibbs description is that the g.f. plays the role of an "independent parameter" indexing the r.p. and r.f.

The one-dimensional case, i.e., the case of r.p., is distinguished from the others: one expects that under general "natural" conditions on the g.f. there exists the unique r.p. corresponding to it. This hypothesis is based on statistical-mechanical arguments: in one-dimensional systems there is no phase transition ${ }^{1}$. The present paper contains a uniqueness theorem for a class of random point processes (r.p.p.). The r.p.p. are associated in statistical mechanics with the states of onedimensional continuous classical systems.

The problem of the uniqueness for r.p.p. was considered in $[6,14,15]$; one of the main restrictions in $[6,14,15]$ was the "hard-core" condition imposed on the g.f. i.e., the assumption that the g.f. takes the value $+\infty$. This assumption simplifies the structure of the r.p.p. associated with the given g.f. and makes possible the use of compactness arguments. In [15] the condition of hard cores is dropped in the case when the g.f. has, in a sense, a compact support. Here we consider the case when the g.f. has, in general, no hard cores and no compact support. The first restriction is replaced by the condition of the non-negativity of the g.f., the second one - by the rapid (super-exponential) decrease of the g.f. at infinity. We must say that our uniqueness theorem is, apparently, far from indicating the precise boundary (in terms of the degree of decrease of the g.f.) between the uniqueness and non-uniqueness of the r.p.p. corresponding to a given g.f. However the necessary conditions for the uniqueness probably are more restrictive in our case than in the case of r.p. with discrete time and a finite single-value space $[4,16]$ or in the case of the r.p.p. corresponding to the g.f. with a hard core $[6,14,15]$.

Theorems 2 and 3 establish some regularity properties of the r.p.p. corresponding to the given g.f.

\section{Random Point Processes and Generating Functions}

The general theory of r.p.p. is developped in [17]. For the reader's convenience we give here some basic definitions. Let $\mathcal{O}$ (resp., $\mathcal{O}(\Omega) \subset \mathcal{O}$ ) be the collection of all finite subsets $\bar{x} \subset R^{1}$ (resp., $\bar{x} \subset \Omega$ where $\Omega \subset R^{1}$ is a bounded Borel set (b.B.s.)) with the point-to-point convergence topology: $\bar{x}_{\alpha} \rightarrow \bar{x}$ iff

a) $n\left(\bar{x}_{\alpha}\right) \rightarrow n(\bar{x})$ where $n(\bar{y})$ is the cardinality (number of the points) of $\bar{y} \in \mathcal{O}$,

b) $\max _{x \in \bar{x}} \min _{x^{\prime} \in \bar{x}_{\alpha}}\left|x-x^{\prime}\right| \rightarrow 0^{2}$.

Denote by $\mathfrak{A}($ resp., $\mathfrak{A}(\Omega) \subset \mathfrak{U})$ the Borel $\sigma$-algebra of subsets of $\mathcal{O}$ (resp., $\mathcal{O}(\Omega)$ ). On $\mathfrak{A}$ (and automatically on $\mathfrak{U}(\Omega)$ ) one defines the measure $\lambda$ :

$$
\lambda(A)=\sum_{n=0}^{\infty} \frac{1}{n !} \operatorname{mes}_{n}\left\{\left(x_{1}, \ldots, x_{n}\right) \in R_{\neq}^{n} \mid\left\{x_{1}, \ldots, x_{n}\right\} \in A\right\},
$$

where $R_{\neq}^{n}=\left\{\left(y_{1}, \ldots, y_{n}\right) \in R^{n}: y_{i} \neq y_{j}, 1 \leqq i<j \leqq n\right\},\left\{x_{1}, \ldots, x_{n}\right\}$ is the unordered set consisting of the points $x_{1}, \ldots, x_{n}$, mes $_{n}$ is the $n$-dimensional Lebesgue measure.

1 In general, this assertion is not true, see $[12,13]$

2 The empty set $\emptyset$ is finite by definition and its cardinality is 0 . In this topology it is an isolated point. We use the same symbol for indicating the empty set as an element of $\mathcal{O}$ or $\mathcal{O}(\Omega)$ and for noting it in a concrete set-theoretical context 
By definition, $\lambda(\emptyset)=1$. Let $f: \mathcal{O} \rightarrow R^{1} \cup\{\infty\}$ be a measurable function, $f(\emptyset)=0$, denote

$$
h_{f}(\bar{x})=\sum_{\bar{z} \subseteq \bar{x}} f(\bar{z}), \bar{x} \in \mathcal{O},
$$

and

$$
h_{f}(\bar{x} \mid \bar{y})=\left\{\begin{array}{l}
\sum_{\substack{z \subseteq x \cup y, z \cap x \neq \phi, z \cap y \neq \phi}} f(\bar{z}) \text { if } \bar{x}=\emptyset, \bar{y} \neq \emptyset \text { and } \bar{x} \cap \bar{y}=\emptyset, \\
0, \text { otherwise. } \\
\bar{x}, \bar{y} \in \mathcal{O} .
\end{array}\right.
$$

Let 2 be the collection of all finite or countable subsets of $R^{1}$ having no limit points in $R^{1}$. The elements of $\mathscr{2}$ are denoted $X, Y$, etc. $\mathscr{Z}$ is provided with the weakest topology in which the map

$$
\pi_{\Omega}: X \in \mathscr{Q} \mapsto X_{\Omega}=X \cap \Omega \in \mathcal{O}(\Omega)
$$

is continuous for any open b.B.s. $\Omega \subset R^{1}$; with this topology 2 is a polish space ${ }^{3}$ [18]. A random point process is an arbitrary probability measure on the Borel $\sigma$-algebra $\mathfrak{B}$ of subsets of 2 .

Another description of the $\sigma$-algebra $\mathfrak{B}$ may be given as follows. Let $\pi_{\Omega}^{-1} A$ be the full pre-image in $\mathscr{Q}$ of a set $A \subset \mathcal{O}(\Omega)$ under the map $\pi_{\Omega}$. The sets $\pi_{\Omega}^{-1} A, A \in \mathfrak{A}(\Omega)$, form a $\sigma$-algebra of subsets of $\mathscr{Q}$ denoted by $\mathfrak{B}_{\Omega}$. Clearly, $\mathfrak{B}_{\Omega_{1}} \subseteq \mathfrak{B}_{\Omega_{2}}$ provided $\Omega_{1} \cong \Omega_{2}$. The union $\bigcup_{\Omega \subset R^{1}, \Omega \text { is b.B.s. }} \mathfrak{B}_{\Omega}$ is denoted $\mathfrak{B} \sim$ it is an algebra of subsets

of $2 . \mathfrak{B}$ is the smallest $\sigma$-algebra containing $\mathfrak{B}^{\sim}$. It two r.p.p., $P_{1}$ and $P_{2}$, coincide on $\mathfrak{B}^{\sim}$, then $P_{1}=P_{2}$ on $\mathfrak{B}$.

Given a r.p.p. $P$, denote $P^{\Omega}$ the probability measure on $\mathfrak{B}_{\Omega}$ induced by $P$ : $P^{\Omega}(A)=P(A), A \in \mathfrak{B}_{\Omega^{\prime}}$. Let $\mathfrak{B}_{\Omega^{c}}$ be the smallest $\sigma$-algebra containing all $\mathfrak{B}_{\Omega^{\prime}}$, where $\Omega^{\prime} \subset R^{1} \backslash \Omega^{4}$. Since 2 is a polish space, for any pair of b.B.s. $\Omega \subseteq \Omega^{\prime} \subset R^{1}$ there exists $P^{\Omega}\left(\cdot \mid \mathfrak{B}_{\Omega^{\prime}}\right)$, the conditional probability measure on $\mathfrak{B}_{\Omega}$, under the $\sigma$-algebra $\mathfrak{B}_{\Omega^{\prime}}$, see [19, p. 53, Theorem 3]. We say that a r.p.p. $P$ is regular (from outside, cf. [4]) if for any b.B.s. $\Omega \subset R^{1}, P$-almost everywhere on $\mathscr{Q}$,

$$
\lim _{N \rightarrow \infty} \operatorname{Var}\left[P^{\Omega}\left(\cdot \mid \mathfrak{B}_{\Omega_{N}^{c}}\right), P^{\Omega}\right]=0,
$$

where $\Omega_{N}=(-N, N), \operatorname{Var}\left[P_{1}^{\Omega}, P_{2}^{\Omega}\right]=\sup _{A \in \mathfrak{B}_{\Omega}}\left|P_{1}^{\Omega}(A)-P_{2}^{\Omega}(A)\right|$ is the variation distance between measures on $\mathfrak{B}_{\Omega}$.

Let $X \in \mathscr{Q}, t \in R^{1}$; define $T_{t} X=\left\{q \in R^{1}: q-t \in X\right\}$. The maps $T_{t}: \mathscr{Q} \rightarrow \mathscr{Q}$ form a one-parameter group of transformations of 2 . We say that a r.p.p. $P$ is invariant if for any $A \in \mathfrak{B}$ and $t \in R^{1}, P\left(T_{t} A\right)=P(A)$. The r.p.p. $P$ is invariant if this equality holds for any $t \in R^{1}$ and $A \in \mathfrak{B}$. Similarly, a function $f$ on $\mathcal{O}$ is called invariant if for any $\bar{x} \in \mathcal{O}$ and $t \in R^{1}, f\left(T_{t} \bar{x}\right)=f(\bar{x})$.

We use below the notation $X_{\Omega}$ for $X \cap \Omega$ in the case of general (not necessary bounded) Borel $\Omega \subset R^{1}$. For $\bar{x} \in \mathcal{O}$ and $X \in \mathscr{Q}$ denote

$$
E_{f}(\bar{x} \mid X)=\lim _{n \rightarrow \infty} \exp \left[-h_{f}\left(\bar{x} \mid X_{\Omega_{N}}\right)\right]
$$

\footnotetext{
3 The topology induced on $\mathcal{O}$ as the subset of $\mathscr{Q}$ does not coincide with the topology of the point-topoint convergence

${ }_{4}$ Here and below $\Omega^{c}$ denotes the complement of $\Omega$ in $R^{1}$; the complement of a set $A$ in $\mathscr{Q}$ is denoted $\bar{A}$
} 
if this limit exists. Let now $\Omega \subset R^{1}$ be a b.B.s., $X \in \mathscr{Q}$; denote

$$
\begin{aligned}
\Xi\left(\Omega, X_{\Omega^{c}}\right) & =\int_{\mathscr{O}(\Omega)} d \lambda(\bar{y}) \exp \left[-h_{f}(\bar{y})\right] E_{f}\left(\bar{y} \mid X_{\Omega^{c}}\right), \\
p_{\Omega}\left(\bar{x} ; X_{\Omega^{c}}\right) & =\Xi\left(\Omega, X_{\Omega^{c}}\right)^{-1} \exp \left[-h_{f}(\bar{x})\right] E_{f}\left(\bar{x} \mid X_{\Omega^{c}}\right),
\end{aligned}
$$

and

$$
P_{\Omega}\left(A ; X_{\Omega^{c}}\right)=\int_{\pi_{\Omega} A} d \lambda(\bar{x}) p_{\Omega}\left(\bar{x} ; X_{\Omega^{c}}\right), A \in \mathfrak{B}_{\Omega},
$$

if the limits and the integrals under consideration exist. We say that a r.p.p. is a solution of the DLR equation ${ }^{5}$ with the g.f. $f$ if for any b.B.s.

a) the limit $E_{f}\left(\bar{x} \mid X_{\Omega^{c}}\right)$ exists for almost all (w.r.t. the measure $\lambda \times P$ ) pairs $(\bar{x}, X) \in \mathcal{O}(\Omega) \times \mathscr{Q}$,

b) the integral $\Xi\left(\Omega, X_{\Omega^{c}}\right)$ converges for almost all (w.r.t. $\left.P\right) X \in \mathscr{Q}$,

c) for any $A \in \mathfrak{B}_{\Omega}$

$$
P(A)=\int_{\mathscr{Q}} P(d X) P_{\Omega}\left(A ; X_{\Omega^{c}}\right) \text {. }
$$

In what follows we consider the functions $f$ satisfying a number of conditions inspired by statistical mechanics. The first assumption is:

(I1) $f$ is an invariant function taking finite values for almost all (w.r.t. $\lambda) \bar{x} \in \mathcal{O}$.

This expresses the absence of hard cores. The next condition is:

(I2) $f(\bar{x}) \equiv 0$ if $n(\bar{x}) \geqq 3, \bar{x} \in \mathcal{Q}$.

This corresponds to the case of pair interactions in statistical mechanics. Conditions (I1) and (I2) mean that $f$ may be identified with a pair $\left(c_{0}, V\right)$ where $c_{0} \in R^{1}$ is a constant, and $V:(0, \infty) \rightarrow R^{1}$ is a measurable function. More precisely, $f(\bar{x})=c_{0}$ if $n(\bar{x})=1$, and $f(\bar{x})=V\left(\left|x-x^{\prime}\right|\right)$ if $h(\bar{x})=2$ and $\bar{x}=\left\{x, x^{\prime}\right\}, x, x^{\prime} \in R^{1}$. In these terms

and

$$
h_{f}(\vec{x})=c_{0} n(\bar{x})+\sum_{x, x^{\prime} \in \bar{x}, x \neq x^{\prime}} V\left(\left|x-x^{\prime}\right|\right), \bar{x} \in \mathcal{O},
$$

$$
h_{f}(\bar{x} \mid \bar{y})=\sum_{z \in \bar{x}, z^{\prime} \in \bar{y}} V\left(\left|z-z^{\prime}\right|\right), \bar{x}, \bar{y} \neq \emptyset, \bar{x} \cap \bar{y}=\emptyset .
$$

Finally,

(I3) $V \geqq 0$ and there exist $d>0$ and a non-increasing positive function $\psi(r)$, $r \geqq d$, such that $\lim _{r \rightarrow \infty} \psi(r) \exp (\alpha r)=0$ for every $\alpha>0$ and $V(r) \leqq \psi(r), r \geqq d$.

Remark. The results formulated below remain valid under some more general assumptions imposed on $h_{f}$, see $\S 2$.

Let $f$ satisfy the conditions (I1-I3). Then for any $\bar{x}, \bar{y} \in \mathcal{O}$ and $X \in \mathscr{Z}$

$$
h_{f}(\bar{x}) \geqq c_{0} n(\bar{x}), h_{f}(\bar{x} \mid \bar{y}) \geqq 0, E_{f}(\bar{x} \mid X) \leqq 1 .
$$

Denoting

$$
\mu(A)=\int_{A} d \lambda(\vec{x}) \exp \left[-c_{0} n(\bar{x})\right], A \subseteq \mathcal{O}, A \in \mathfrak{U},
$$

5 See Dobrushin [3-6], Lanford and Ruelle [7], Ruelle [8] 
we have the following estimates:

$$
\begin{aligned}
1 & =\exp \left[-h_{f}(\emptyset)\right] E_{f}\left(\emptyset \mid X_{\Omega^{c}}\right) \leqq \Xi\left(\Omega, X_{\Omega^{c}}\right) \\
& \leqq \mu(O(\Omega))=\exp \left(e^{c_{0}} \operatorname{mes}_{1} \Omega\right) .
\end{aligned}
$$

Hence, for any b.B.s. $\Omega \subset R^{1}$ and $X \in \mathscr{Q}, P_{\Omega}\left(\cdot ; X_{\Omega^{c}}\right)$ defines a probability measure on $\mathfrak{B}_{\Omega}$. Let $A \in \mathfrak{B}_{\Omega^{2}}$. As the function of $X, P_{\Omega}\left(A ; X_{\Omega^{c}}\right)$ is $\mathfrak{B}_{\Omega^{c}}$-measurable. Hence, if $P$ is an arbitrary solution of the DLR equation with the g.f. $f$, then $P_{\Omega}\left(\cdot ; X_{\Omega^{c}}\right)$ coincides, for $P$-almost all $X \in \mathscr{Q}$, with the restriction on $\mathfrak{B}_{\Omega}$ of the conditional probability measure $P\left(\cdot \mid \mathfrak{B}_{\Omega^{c}}\right)(X)$ generated by $P$.

We conclude this section by a simple lemma whose proof is omitted.

Lemma 1.1. Suppose $\Omega_{1} \subset R^{1}$ and $\Omega_{2} \subset R^{1}$ are b.B.s., $\Omega_{1} \cap \Omega_{2}=\emptyset$. The map

$$
\mathcal{O}\left(\Omega_{1} \cup \Omega_{2}\right) \ni \bar{x} \leftrightarrow\left(\bar{x} \cap \Omega_{1}, \bar{x} \cap \Omega_{2}\right) \in \mathcal{O}\left(\Omega_{1}\right) \times \mathcal{O}\left(\Omega_{2}\right)
$$

is an isomorphism between the measure spaces $\left(\mathcal{O}\left(\Omega_{1} \cup \Omega_{2}\right), \mathfrak{A}\left(\Omega_{1} \cup \Omega_{2}\right), \lambda\right)$ and $\left(\mathcal{O}\left(\Omega_{1}\right), \mathfrak{U}\left(\Omega_{1}\right), \lambda\right) \times\left(\mathcal{O}\left(\Omega_{2}\right), \mathfrak{Z}\left(\Omega_{2}\right), \lambda\right)$.

As the consequence of Lemma 1.1, we obtain that if $P$ is a solution of the $D L R$ equation with the $g . f . f$ and $\Omega \subset \tilde{\Omega} \subset R^{1}$ are b.B.s., then for any $A \in \mathfrak{B}_{\Omega}$

where

$$
P(A)=\int_{\mathscr{2}} P(d X) P_{\tilde{\Omega}}^{\Omega}\left(A ; X_{\tilde{\Omega}^{c}}\right),
$$

$$
\begin{aligned}
& P_{\tilde{\Omega}}^{\Omega}\left(A ; X_{\tilde{\Omega}^{c}}\right)=\int_{\pi_{\Omega} A} d \lambda(\bar{x}) p_{\tilde{\Omega}}^{\Omega}\left(\bar{x} ; X_{\tilde{\Omega}^{c}}\right) \\
& p_{\tilde{\Omega}}^{\Omega}\left(\bar{x} ; X_{\tilde{\Omega}^{c}}\right)=\Xi\left(\tilde{\Omega}, X_{\tilde{\Omega}^{c}}\right)^{-1} \Xi_{\Omega}\left(\bar{x}, \tilde{\Omega} ; X_{\tilde{\Omega}^{c}}\right)
\end{aligned}
$$

and

$$
\Xi_{\Omega}\left(\bar{x}, \tilde{\Omega} ; X_{\tilde{\Omega}^{c}}\right)=\int_{\mathcal{O}(\tilde{\Omega} \backslash \Omega)} d \lambda(\bar{y}) \exp \left[-h_{f}(\bar{x} \cup \bar{y})\right] E_{f}\left(\bar{x} \cup \bar{y} \mid X_{\tilde{\Omega}^{c}}\right) .
$$

In other terms, $P_{\tilde{\Omega}}^{\Omega}\left(\cdot ; X_{\tilde{\Omega}^{c}}\right)$ coincides, for $P$-almost all $X \in \mathcal{Q}$, with the restriction on $\mathfrak{B}_{\Omega}$ of $P\left(\cdot \mid \mathfrak{B}_{\tilde{\Omega}^{c}}\right)(X)$.

\section{Formulation of Results and Preliminary Estimates}

We prove the following theorems.

Theorem 1. Let $f$ obey $(I 1-I 3)$. There exists a unique solution of the DLR equation with the g.f. $f$.

Theorem 2. The solution of the DLR equation with the g.f. $f$ obeying (I1-I3) is a regular r.p.p.

Theorem 3. The solution $P$ of the DLR equation with the g.f. $f$ obeying (I1-I3) is an invariant r.p.p. The triple $\left(\mathscr{Q}, P,\left\{T_{t}, t \in R^{1}\right\}\right)$ is a generalized $B$-flow in the sense of ergodic theory (see [20]).

Remarks. a) The statements of Theorems 1-3 remain true under more general conditions. In addition to (I1) it is sufficient to assume that,

( $\left.\mathrm{I}^{\prime} 1\right)$ there exist constants $c_{1} \in R^{1}$ and $c_{2} \geqq 0$ such that

$$
h_{f}(\bar{x}) \geqq c_{1} n(\bar{x}), \bar{x} \in \mathcal{O} \text {, }
$$


and

$h_{f}(\bar{x} \mid \bar{y}) \geqq-c_{2} \min [n(\bar{x}), n(\bar{y})], \bar{x} \subset(-\infty, 0), \bar{y} \subset[0, \infty)$,

$\left(\mathrm{I}^{\prime} 2\right)$ there exist a constant $d>0$ and a non-increasing function $\psi:[d, \infty) \rightarrow R^{1}$ such that $\lim _{r \rightarrow \infty} \psi(r) \exp (\alpha r)=0$ for every $\alpha>0$, and for any $\bar{x} \subset(-\infty, 0), \bar{y} \subset[d, \infty)$,

$$
\left|h_{f}(\bar{x} \mid \bar{y})\right| \leqq \sum_{i, j \in \mathbb{Z}_{1}} \psi(|i-j|) n(\bar{x}, i) n(\bar{y}, j)
$$

where $n(\bar{x}, i)=n(\bar{x} \cap[i, i+1)), n(\bar{y}, j)=n(\bar{y} \cap[j, j+1))$.

If we assume that $f(\bar{x}) \equiv 0$ when $\operatorname{diam} \bar{x}=\max _{x, x^{\prime} \in \bar{x}}\left|x-x^{\prime}\right|>d_{1}$ (the finite range or compact support assumption), then it is possible to prove statements like Theorems 1-3 for superstable g.f. [8] dropping the condition

$$
h_{f}(\bar{x} \mid \bar{y}) \geqq-c_{2} \min [n(\bar{x}), n(\bar{y})] .
$$

b) Similar results may be obtained for marked r.p.p. and r.p. with the discrete time with non-compact single-value space. We do not dwell here on this problem.

Before going to the proof of Theorem 1 we formulate a simple lemma playing an important role in our analysis.

Lemma 2.1. Let $\psi:[d, \infty) \rightarrow R^{1}$ be a function satisfying the conditions (I3), $d_{1}>0$ and $d_{2}>0$ be fixed numbers. Then one can and

i) find two increasing sequences $\left\{r_{n}, n \geqq 1\right\}$ and $\left\{s_{n}, n \geqq 1\right\}$ of positive integers,

ii) find a (double-indexed) sequence $\left\{m_{j}^{(n)}, j \geqq 0, n \geqq 1\right\}$ of positive integers such that $m_{0}^{(n)} \leqq m_{1}^{(n)} \leqq \ldots$ and,

$$
\begin{aligned}
& \text { I } \sum_{n=1}^{\infty} a_{n}^{(1)}\left(d_{1}\right)<\infty \text { where } a_{n}^{(1)}\left(d_{1}\right)=\sum_{j=1}^{\infty} \exp \left(-d_{1} m_{j}^{(n)} \ln m_{j}^{(n)}\right), \\
& \text { II } \lim _{n \rightarrow \infty} a_{n}^{(2)}\left(d_{1}\right)=0 \text { where } a_{n}^{(2)}\left(d_{1}\right)=\left[r_{n}\left(s_{n}+1\right)+1\right] \exp \left(-d_{1} m_{0}^{(n)} \ln m_{0}^{(n)}\right), \\
& \text { III } \lim _{n \rightarrow \infty} a_{n}^{(3)}=0 \text { where } a_{n}^{(3)}=2\left(m_{0}^{(n)}\right)^{2}\left[r_{n}\left(s_{n}+1\right)+1\right] \sum_{k \geqq r_{n}} \psi(k) \\
& \quad+2 m_{0}^{(n)} \sum_{k \geqq r_{n}+1} \psi(k) \sum_{l=1}^{k-r_{n}} m_{l}^{(n)}+\sum_{k \geqq r_{n}\left(s_{n}+1\right)} \psi(k) \sum_{l=1}^{k-r_{n}\left(s_{n}+1\right)} m_{l}^{(n)} m_{k-r_{n}\left(s_{n}+1\right)-l}^{(n)} \sum_{l=1} \ln a_{n}^{(4)}\left(d_{2}\right)=s_{n} \exp \left(-2 d_{2} r_{n}\right) \\
& \text { IV } \lim _{n \rightarrow \infty} a_{n}^{(4)}\left(d_{2}\right)=\infty \text { where } a^{(n)} m_{j}^{(n)}-d_{2}\left(r_{n}+1\right) .
\end{aligned}
$$

Proof of Lemma 2.1. It suffices to take $s_{n}=\left[a^{n}\right]$ where $a>1$ is large enough and then to think

$$
r_{n}=\left[\left(2 d_{2}+b\right)^{-1} \ln s_{n}\right], m_{0}^{(n)}=\left[\ln s_{n}\right], m_{j}^{(n)}=m_{j-1}^{(n)}+1, j=1.2, \ldots,
$$

where $b>0$ is a fixed number, [] denotes the integer part.

Now we start with the proof of Theorem 1. In the process of proving we establish some auxiliary Lemmas used as well in the proof of Theorems 2 and 3. In this section we state preliminary estimates; the proof will be completed in Section 3. 
Proof of Theorem 1. The existence of a solution of the DLR equation with the g.f. $f$ follows from Theorem 5.5 in $[8]^{6}$. Hence there is only to prove the uniqueness. We begin with some auxiliary constructions. We suppose once and for all that $d>0$ in (I3) is chosen $\leqq 1$, otherwise it would ne necessary to make some obvious changes in the arguments below. Let $m=1,2, \ldots$; denote

and

$$
\mathcal{O}_{m}=\{\bar{x} \in \mathcal{O}([0,1)): n(\bar{x})=m\}, \mathcal{O}_{>m}=\bigcup_{k>m} \mathcal{O}_{k}, \mathcal{O}_{\leqq m}=\bigcup_{k \leqq m} \mathcal{O}_{k},
$$

$$
\mathscr{Q}_{>m}=\pi_{[0,1)}^{-1} \mathcal{O}_{>m}, \mathscr{Q}_{\leqq m}=\pi_{[0,1)}^{-1} \mathcal{O}_{\leqq m} .
$$

Let $\mathrm{P}$ be a solution of the DLR equation with the g.f. $f$. Using (*) we obtain

$$
P\left(\mathscr{Q}_{>m}\right) \leqq \mu\left(\mathcal{O}_{>m}\right)=\sum_{m^{\prime}>m} \frac{1}{m^{\prime} !} \exp \left(-c_{0} m^{\prime}\right)<\exp \left(-d_{1} m \ln m\right),
$$

where $d_{1}>0$ depends on $c_{0}$ but does not depend on $m$; in what follows we take it as the argument value for $a_{n}^{(1)}$ and $a_{n}^{(2)}$. The same estimate holds for $P\left(T_{k} \mathscr{Q}_{>m}\right)$, $k= \pm 1, \pm 2, \ldots$. If now

$$
\mathscr{Q}_{+}^{(n)}=\bigcap_{k=0}^{\infty} T_{k^{2}} \mathscr{Q}_{\leqq m_{k+1}^{(n)}}, \mathscr{Q}_{-}^{(n)}=\bigcap_{k=1}^{\infty} T_{-k^{2}} \mathscr{Q}_{\leqq m^{(n)}},
$$

then for the complements, $\overline{\mathscr{Z}}_{+}^{(n)}$ and $\overline{\mathscr{Q}}_{-}^{(n)}$, we have the estimates

$$
P\left(\overline{\mathscr{Q}}_{+}^{(n)}\right), P\left(\overline{\mathscr{Q}}_{-}^{(n)}\right)<a_{n}^{(1)}\left(d_{1}\right) \text {. }
$$

Let $0<\varepsilon<1$ be an arbitrary number. Our aim is to prove that if r.p.p. $P^{\prime}$ and $P^{\prime \prime}$ are solutions of the DLR equation with the g.f. $f$ then for any b.B.s. $\Omega \subset R^{1}$ and $A \in \mathfrak{B}_{\Omega},\left|P^{\prime}(A)-P^{\prime \prime}(A)\right|<\varepsilon$. In what follows we think $d_{1}$ is fixed as it is indicated above and $d_{2}=2 e^{-c_{0}}$ and omit these symbols from the notations. We omit also the index $f$ in $h_{f}$ and $h_{f}(\cdot \mid \cdot)$. Fix sequences $\left\{r_{n}\right\},\left\{s_{n}\right\}$ and $\left\{m_{j}^{(n)}\right\}$ satisfying the relations I-IV of Lemma 2.1. Given $0<\varepsilon<1$, choose $n$ so large that the following inequalities hold:

$$
a_{n}^{(1)}, a_{n}^{(2)}<1 / 84 \varepsilon, \max \left[e^{2 a_{n}^{(3)}}-1,1-e^{-2 a_{n}^{(3)}}\right]<1 / 6 \varepsilon, e^{-a_{n}^{(4)}}<1 / 56 \varepsilon .
$$

Now fix some b.B.s. $\Omega \subset R^{1}$. Without loss of generality we suppose that $\Omega=\left[\alpha_{1}, \alpha_{2}\right)$ where $\alpha_{1}<\alpha_{2}$ are integers. For $A \in \mathfrak{B}_{\Omega}$ denote

\section{Denote}

$$
A^{-+}(\Omega)=\left(\pi_{\Omega} A\right) \cap\left(\bigcap_{k=0}^{\alpha_{2}-\alpha_{1}-1} T_{\alpha_{1}+k} \mathcal{O}_{\leqq}\right) \cap\left(\bigcap_{k=1}^{\alpha_{2}-\alpha_{1}} T_{\alpha_{2}-k} \mathcal{O}_{\leqq} m_{k}^{(n)}\right) .
$$

$$
\begin{aligned}
n_{1} & =n_{1}(n)=r_{n}\left(s_{n}+1\right)+1, \\
\Omega^{-} & =\Omega^{-}(n)=\left[\alpha_{1}-n_{1}, \alpha_{1}\right), \Omega^{+}=\Omega^{+}(n)=\left[\alpha_{2}, \alpha_{2}+n_{1}\right), \Omega^{\prime}=\Omega^{-} \cup \Omega \cup \Omega^{+},
\end{aligned}
$$

and

$$
\begin{aligned}
& \mathcal{O}^{(n)}\left(\Omega^{-}\right)=\mathcal{O}\left(\Omega^{-}\right) \cap\left(\bigcap_{k=\alpha_{1}-n_{1}}^{\alpha_{1}-1} T_{k} \mathcal{O}_{\leqq} m_{0}^{(n)}\right), \\
& \mathcal{O}^{(n)}\left(\Omega^{+}\right)=\mathcal{O}\left(\Omega^{+}\right) \cap\left(\bigcap_{k=\alpha_{2}}^{\alpha_{2}+n_{1}-1} T_{k} \mathcal{O}_{\leqq} m_{0}^{(n)}\right) .
\end{aligned}
$$

6 Formally, Theorem 5.5 in [8] is stated for the case of superstable pair potentials [a sufficient condition for the superstability is: $V \in C(0, \infty), V \geqq 0$ and $\lim V(r)>0]$, but the method of the proof may be extended to our case without difficulties 
Let

$$
\begin{aligned}
& \Xi_{n}^{(1)}\left(\Omega^{\prime} ; X_{\Omega^{\prime c}}\right) \\
& =\int_{\mathscr{Q}^{-+}(\Omega)} d \lambda(\bar{y}) \int_{\mathcal{O}^{(n)}\left(\Omega^{-}\right)} d \lambda\left(\bar{y}^{-}\right) \int_{\mathcal{O}^{(n)}\left(\Omega^{+}\right)} d \lambda\left(\bar{y}^{+}\right) \exp \left[-h\left(\bar{y} \cup \bar{y}^{-} \cup \bar{y}^{+}\right)\right] \\
& \quad \cdot E\left(\bar{y} \cup \bar{y}^{-} \cup \bar{y}^{+} \mid X_{\Omega^{\prime c}}\right) \\
& \Xi_{\Omega^{\prime}, n}^{(1)}\left(\bar{x}, \Omega^{\prime} ; X_{\Omega^{\prime} c}\right) \\
& =\int_{\mathcal{O}\left(\Omega^{(n)}\left(\Omega^{-}\right)\right.} d \lambda\left(\bar{y}^{-}\right) \int_{\mathcal{O}^{(n)}\left(\Omega^{+}\right)} d \lambda\left(\bar{y}^{+}\right) \exp \left[-h\left(\bar{x} \cup \bar{y}^{-} \cup \bar{y}^{+}\right)\right] E\left(\bar{x} \cup \bar{y}^{-} \cup \bar{y}^{+} \mid X_{\Omega^{\prime c}}\right) \\
& \left(p_{\Omega^{\prime}}^{\Omega}\right)_{n}^{(1)}\left(\bar{x} ; X_{\Omega^{\prime} c}\right)=\Xi_{n}^{(1)}\left(\Omega^{\prime} ; X_{\Omega^{\prime} c}\right)^{-1} \Xi_{\Omega, n}^{(1)}\left(\bar{x}, \Omega^{\prime} ; X_{\Omega^{\prime} c}\right) .
\end{aligned}
$$

Denote

$$
\left(P_{\Omega^{\prime}}^{\Omega}\right)_{n}^{(1)}\left(A ; X_{\Omega^{\prime c}}\right)=\int_{A^{-+}(\Omega)} d \lambda(\bar{x})\left(p_{\Omega^{\prime}}^{\Omega}\right)_{n}^{(1)}\left(\bar{x} ; X_{\Omega^{\prime} c}\right)
$$

and

$$
P_{n}^{(1)}(A)=\int_{T_{\alpha_{1}-n_{1}-1} \mathscr{Q}_{-}^{(n)} \cap T_{\alpha_{2}+n_{1}} \mathscr{Q}_{+}^{(n)}} P(d X)\left(P_{\Omega^{\prime}}^{\Omega}\right)_{n}^{(1)}\left(A ; X_{\Omega^{\prime} c}\right) .
$$

Lemma 2.2. Let $P$ satisfy the DLR equation with the g.f. $f$. Then for any $A \in \mathfrak{B}_{\Omega}$ $\left|P(A)-P_{n}^{(1)}(A)\right|<1 / 6 \varepsilon$.

Proof of Lemma 2.2. According to Lemma 2.1, the choice of $n$ and the estimate for $P\left(\overline{\mathscr{Q}}_{\overline{+}}^{(n)}\right)$,

$$
0 \leqq P(A)-\int_{T_{\alpha_{1}-n_{1}-1} \mathscr{2}_{\left(n^{\prime}\right)} \cap T_{\alpha_{2}+n_{1} 2_{+}^{(n)}}} P(d X) P\left(A ; X_{\Omega^{\prime c}}\right) \leqq 2 / 84 \varepsilon=1 / 42 \varepsilon .
$$

Analogous arguments show that

$$
\left|P\left(A ; X_{\Omega^{\prime c}}\right)-\int_{A^{-+}(\Omega)} d \lambda(\bar{x}) p_{\Omega^{\prime}}^{\Omega}\left(\bar{x} ; X_{\Omega^{\prime} c}\right)\right| \leqq 1 / 42 \varepsilon
$$

and hence

$$
\int_{T_{\alpha_{1}-n_{1}-1} \mathscr{2}_{-}^{(n)} \cap T_{\alpha_{2}+n_{1}} 2_{+}^{(n)}} P(d X)\left|P\left(A ; X_{\Omega^{\prime c}}\right)-\int_{A^{-}(\Omega)} d \lambda(\bar{x}) p_{\Omega^{\prime}}^{\Omega}\left(\bar{x} ; X_{\Omega^{\prime} c}\right)\right|<1 / 42 \varepsilon .
$$

Furthermore,

$$
\begin{aligned}
& 0 \leqq 1-\Xi\left(\Omega^{\prime} ; X_{\Omega^{\prime c}}\right)^{-1} \Xi_{n}^{(1)}\left(\Omega^{\prime} ; X_{\Omega^{\prime} c}\right) \\
& =\Xi\left(\Omega^{\prime} ; X_{\Omega^{\prime} c}\right)^{-1}\left[\Xi\left(\Omega^{\prime} ; X_{\Omega^{\prime} c}\right)-\Xi_{n}^{(1)}\left(\Omega^{\prime} ; X_{\Omega^{\prime} c}\right)\right] .
\end{aligned}
$$

The difference in the square brackets may be expressed as the sum of three integrals

$$
\begin{aligned}
& {\left[\int_{\mathcal{O}(\Omega) \backslash \mathscr{Q}^{-+}(\Omega)} d \lambda(y) \int_{\mathcal{O}\left(\Omega^{-}\right)} d \lambda\left(\bar{y}^{-}\right) \int_{\mathcal{O}\left(\Omega^{+}\right)} d \lambda\left(\bar{y}^{+}\right)+\int_{\mathscr{Q}^{-+}(\Omega)} d \lambda(\bar{y}) \int_{\mathcal{O}\left(\Omega^{-}\right) \backslash \mathcal{O}^{(n)}\left(\Omega^{-}\right)} d \lambda\left(\bar{y}^{-}\right)\right.} \\
& \times \int_{\mathcal{O}\left(\Omega^{+}\right)} d \lambda\left(\bar{y}^{+}\right) \\
& \left.+\int_{\mathscr{Q}^{-+}(\Omega)} d \lambda(\bar{y}) \int_{\mathcal{O}(n)\left(\Omega^{-}\right)} d \lambda\left(\bar{y}^{-}\right) \int_{\mathcal{O}\left(\Omega^{+}\right) \backslash \mathcal{O}^{(n)}\left(\Omega^{+}\right)} d \lambda\left(\bar{y}^{+}\right)\right] \exp \left[-h\left(\bar{y} \cup \bar{y}^{-} \cup \bar{y}^{+}\right)\right] \\
& \times E\left(\bar{y} \cup \bar{y}^{-} \cup \bar{y}^{+}\left(X_{\Omega^{\prime}}\right) .\right.
\end{aligned}
$$


All of them are estimated similarly and do not exceed, respectively, $2 a_{n}^{(1)}, a_{n}^{(2)}$ and $a_{n}^{(2)}$, times $\Xi\left(\Omega^{\prime}, X_{\Omega^{\prime}}\right)$. For instance, the second integral is less then

$$
\begin{aligned}
& \sum_{k=\alpha_{1}-n_{1}}^{\alpha_{1}-1} \int_{\mathcal{O}\left(\Omega^{\prime} \backslash[k, k+1)\right)} d \lambda(\bar{y}) \exp [-h(\bar{y})] E\left(\bar{y} \mid X_{\Omega^{\prime c}}\right) \mu\left(\mathcal{O}_{>m_{0}^{(n)}}\right) \\
& \leqq\left[\left(s_{n}+1\right) r_{n}+1\right] \exp \left(-d_{1} m_{0}^{(n)} \ln m_{0}^{(n)}\right) \Xi\left(\Omega^{\prime} ; X_{\Omega^{\prime} c}\right) ;
\end{aligned}
$$

we have used here Lemma 1.1 and the estimates $(*)$. Finally, we obtain

$$
0 \leqq 1-\Xi\left(\Omega^{\prime} ; X_{\Omega^{\prime}}\right)^{-1} \Xi_{n}^{(1)}\left(\Omega^{\prime} ; X_{\Omega^{\prime} c}\right) \leqq 4 / 84 \varepsilon=1 / 21 \varepsilon,
$$

and hence,

$$
0 \leqq \Xi_{n}^{(1)}\left(\Omega^{\prime} ; X_{\Omega^{\prime} c}\right)^{-1} \Xi\left(\Omega^{\prime} ; X_{\Omega^{\prime} c}\right)-1 \leqq 1 / 21 \varepsilon(1-1 / 21 \varepsilon)^{-1}<2 / 21 \varepsilon .
$$

Now we estimate

$$
\begin{aligned}
& \left|\int_{A^{-+}(\Omega)} d \lambda(\bar{x})\left[p_{\Omega^{\prime}}^{\Omega}\left(\bar{x} ; X_{\Omega^{\prime} c}\right)-\left(p_{\Omega^{\prime}}^{\Omega}\right)_{n}^{(1)}\left(\bar{x} ; X_{\Omega^{\prime} c}\right)\right]\right| \\
& \leqq \Xi\left(\Omega^{\prime} ; X_{\Omega^{\prime} c}\right)^{-1}\left(\left|\int_{A^{-+}(\Omega)} d \lambda(\bar{x})\left[\Xi_{\Omega}\left(\bar{x}, \Omega^{\prime} ; X_{\Omega^{\prime} c}\right)-\Xi_{\Omega, n}^{(1)}\left(\bar{x}, \Omega^{\prime} ; X_{\Omega^{\prime}}\right)\right]\right|\right. \\
& \left.+2 / 21 \varepsilon \int_{A^{-+}(\Omega)} d \lambda(\bar{x}) \Xi_{\Omega, n}^{(1)}\left(\bar{x}, \Omega^{\prime} ; X_{\Omega^{\prime} c}\right)\right) .
\end{aligned}
$$

The integral under the absolute value sign in the right hand side (RHS) of the last inequality may be estimated like $\left[\Xi\left(\Omega^{\prime} ; X_{\Omega^{\prime} c}\right)-\Xi_{n}^{(1)}\left(\Omega^{\prime} ; X_{\Omega^{\prime}}\right)\right]$ above and is less than $2 a_{n}^{(2)} \Xi\left(\Omega^{\prime} ; X_{\Omega^{\prime}}\right)$. Hence, the RHS of the inequality does not exceed $5 / 42 \varepsilon$. We obtain that

$$
\left|\int_{T_{\alpha_{1}-n_{1}-1} \mathscr{2}_{\left(n^{\prime}\right)} \cap T_{\alpha_{2}+n_{1}} \mathscr{2}_{+}^{(n)}} P(d X) \int_{A^{-+}(\Omega)} d \lambda(\bar{x}) p_{\Omega^{\prime}}^{\Omega}\left(\bar{x} ; X_{\Omega^{\prime} \mathrm{c}}\right)-P_{n}^{(1)}(A)\right|<5 / 42 \varepsilon,
$$

and on account of preceding bounds, $\left|P(A)-P_{n}^{(1)}(A)\right|<1 / 6 \varepsilon$. Q.E.D.

The next step is to introduce the "cutoff" function $f_{n}(\bar{x})$ associated with the pair $\left(c_{0}, V_{n}\right)$ where $V_{n}(r)=V(r)$ if $0<r<r_{n}$ and $V_{n}(r)=0$ if $r \geqq r_{n}$. Hence,

and

$$
h_{n}(\bar{x})=h_{f_{n}}(\bar{x})=c_{0} n(\bar{x})+\sum_{\substack{x, x^{\prime} \in \bar{x}, 0<\left|x-x^{\prime}\right|<r_{n}}} V\left(\left|x-x^{\prime}\right|\right), \bar{x} \in \mathcal{O},
$$

$$
h_{n}(\bar{x} \mid \vec{y})=h_{f_{n}}(\bar{x} \mid \vec{y})=\sum_{\substack{z \in \bar{x}, z^{\prime} \in \bar{y}, 0<\left|z-z^{\prime}\right|<r_{n}}} V\left(\left|z-z^{\prime}\right|\right), \bar{x}, \bar{y} \neq \emptyset, \bar{x} \cap \bar{y}=\emptyset .
$$

Denote $E_{n}(\bar{x} \mid X)=E_{f_{n}}(\bar{x} \mid X)$,

$$
\begin{aligned}
& \Xi_{n}^{(2)}\left(\Omega^{\prime} ; X_{\Omega^{\prime c}}\right)=\int_{\mathscr{Q}^{-+}(\Omega)} d \lambda(\bar{y}) \int_{\mathcal{O}(n)\left(\Omega^{-}\right)} d \lambda\left(\bar{y}^{-}\right) \int_{\mathcal{O}(n)\left(\Omega^{+}\right)} d \lambda\left(\bar{y}^{+}\right) \exp [-h(\bar{y})] \\
& \cdot \exp \left[-h_{n}(\bar{y})-h_{n}\left(\bar{y} \mid \bar{y}^{-} \cup \bar{y}^{+}\right)\right] E_{n}\left(\bar{y}^{-} \cup \bar{y}^{+} \mid X_{\Omega^{\prime} c}\right), \\
& \Xi_{\Omega, n}^{(2)}\left(\bar{x}, \Omega^{\prime} ; X_{\Omega^{\prime}}\right)=\int_{\left(\mathcal{O}^{(n)}\left(\Omega^{-}\right)\right.} d \lambda\left(\bar{y}^{-}\right) \int_{\mathcal{O}(n)\left(\Omega^{+}\right)} d \lambda\left(\bar{y}^{+}\right) \exp [-h(\bar{x})] \\
& \cdot \exp \left[-h_{n}\left(\bar{y}^{-}\right)-h_{n}\left(\bar{y}^{+}\right)-h_{n}\left(\bar{y} \mid \bar{y}^{-} \cup \bar{y}^{+}\right)\right] E_{n}\left(\bar{y}^{-} \cup \bar{y}^{+} \mid X_{\Omega^{\prime} c}\right), \\
& \left(p_{\Omega^{\prime}}^{\Omega}\right)_{n}^{(2)}\left(\bar{x} ; X_{\Omega^{\prime}}\right)=\Xi_{n}^{(2)}\left(\Omega^{\prime} ; X_{\Omega^{\prime} c}\right) \Xi_{\Omega_{n}, n}^{(2)}\left(\bar{x}, \Omega^{\prime} ; X_{\Omega^{\prime c}}\right) .
\end{aligned}
$$

Denote

$$
\left(P_{\Omega^{\prime}}^{\Omega}\right)_{n}^{(2)}\left(A ; X_{\Omega^{\prime c}}\right)=\int_{A^{-+}(\Omega)} d \lambda(\bar{x})\left(p_{\Omega^{\prime}}^{\Omega}\right)_{n}^{(2)}\left(\bar{x} ; X_{\Omega^{\prime} c}\right)
$$


and

$$
P_{n}^{(2)}(A)=\int_{T_{\alpha_{1}-n_{1}-1} \mathscr{Q}_{(n)}^{(n)} \cap T_{\alpha_{2}+n_{1}} \mathscr{2}_{+}^{(n)}} P(d X)\left(P_{\Omega^{\prime}}^{\Omega}\right)_{n}^{(2)}\left(A ; X_{\Omega^{\prime} c}\right) .
$$

Lemma 2.3. Let $P$ satisfy the $D L R$ equation with the g.f. $f$. Then for any $A \in \mathfrak{B}_{\Omega}$

$$
\left|P_{n}^{(1)}(A)-P_{n}^{(2)}(A)\right|<1 / 6 \varepsilon .
$$

Proof of Lemma 2.3. Suppose $\bar{y} \in \mathscr{Q}^{-+}(\Omega), \bar{y}^{-} \in \mathcal{O}^{(n)}\left(\Omega^{-}\right), \bar{y}^{+} \in \mathcal{O}^{(n)}\left(\Omega^{+}\right)$and $X \in T_{\alpha_{1}-n_{1}-1} \mathscr{Q}_{-}^{(n)} \cap T_{\alpha_{2}+n_{1}} \mathscr{Q}_{+}^{(n)}$. A direct calculation shows that

$$
\begin{aligned}
1 \leqq & \frac{\exp \left[-h(\bar{y})-h_{n}\left(\bar{y}^{-}\right)-h_{n}\left(\bar{y}^{+}\right)-h_{n}\left(\bar{y} \mid \bar{y}^{-} \cup \bar{y}^{+}\right)\right] E_{n}\left(\bar{y}^{-} \cup \bar{y}^{+} \mid X_{\Omega^{\prime} c}\right)}{\exp \left[-h\left(\bar{y} \cup \bar{y}^{-} \cup \bar{y}^{+}\right)\right] E\left(\bar{y} \cup \bar{y}^{-} \cup \bar{y}^{+} \mid X_{\Omega^{\prime c}}\right.} \\
& \leqq \exp \left[4\left(m_{0}^{(n)}\right)^{2} \sum_{k \geqq r_{n}} \psi(k)+4 m_{0}^{(n)} \sum_{k>r_{n}} \psi(k) \sum_{l=1}^{k-r_{n}} m_{l}^{(n)}\right. \\
& \left.+2 \sum_{k>n_{1}} \psi(k) \sum_{l=1}^{k-n_{1}} m_{l}^{(n)} m_{k-n_{1}+1-l}^{(n)}\right]
\end{aligned}
$$

Hence:

$$
\begin{aligned}
& 1 \leqq \frac{\Xi_{n}^{(2)}\left(\Omega^{\prime} ; X_{\Omega^{\prime} c}\right)}{\Xi_{n}^{(1)}\left(\Omega^{\prime} ; X_{\Omega^{\prime} c}\right)}, \frac{\Xi_{\Omega, n}^{(2)}\left(\bar{x}, \Omega^{\prime} ; X_{\Omega^{\prime} c}\right)}{\Xi_{\Omega, n}^{(1)}\left(\bar{x}, \Omega^{\prime} ; X_{\Omega^{\prime} c}\right)} \leqq \exp \left(2 a_{n}^{(3)}\right), \bar{x} \in \mathscr{Q}^{-+}(\Omega), \\
& \left|\left(P_{\Omega^{\prime}}^{\Omega}\right)_{n}^{(1)}\left(A ; X_{\Omega^{\prime} c}\right)-\left(P_{\Omega^{\prime}}^{\Omega}\right)_{n}^{(2)}\left(A ; X_{\Omega^{\prime} c}\right)\right|<\max \left[e^{2 a_{n}^{(3)}}-1,1-e^{-2 a_{n}^{(3)}}\right],
\end{aligned}
$$

and, according to $(* *),\left|P_{n}^{(1)}(A)-P_{n}^{(2)}(A)\right|<1 / 6 \varepsilon$. Q.E.D.

\section{End of the Proof of Theorem 1}

Denote $L_{n}=\left[-r_{n}, r_{n}\right)$,

$$
\mathcal{O}^{(n)}\left(L_{n}\right)=\mathcal{O}\left(L_{n}\right) \cap\left(\bigcap_{k=-r_{n}}^{r_{n}-1} T_{k} \mathcal{O} \leqq m_{0}^{(n)}\right)
$$

and $\mathscr{L}_{n}^{2}=\mathscr{L}^{2}\left(\mathcal{O}^{(n)}\left(L_{n}\right), \lambda\right) .\langle$,$\rangle denotes below the inner product in \mathscr{L}_{n}^{2}$. Let

$$
\mathscr{K}_{n}(\bar{x}, \bar{y})=\exp \left[-h\left(\bar{x}_{-}\right)-h\left(\bar{x}_{+}\right)-h_{n}\left(T_{-r_{n}} \bar{x}_{-} \mid \bar{y}\right)-h_{n}\left(T_{r_{n}} \bar{x}_{+} \mid \bar{y}\right)\right]
$$

where $\bar{x}_{-}=\bar{x} \cap\left[-r_{n}, 0\right), \quad \bar{x}_{+}=\bar{x} \cap\left[0, r_{n}\right), \bar{x}, \bar{y} \in \mathcal{O}^{(n)}\left(L_{n}\right)$. Consider the operator $K_{n}$ in $\mathscr{L}_{n}^{2}$ given by

$$
\left(K_{n} F\right)(\bar{x})=\int_{\mathcal{O}(n)\left(L_{n}\right)} d \lambda(\bar{y}) \mathscr{K}_{n}(\bar{x}, \bar{y}) F(\bar{y}) .
$$

Using the inequalities (*) (which hold, of course, for $h_{n}$ as well as for $h$ ), it is easy to check that $K_{n}$ is a Hilbert-Schmidt operator in $\mathscr{L}_{n}^{2}$. Since $\mathscr{K}_{n}(\bar{x}, \bar{y}) \geqq 0$, it preserves the cone of positive functions in $\mathscr{L}_{n}^{2}$.

Denote

$$
\begin{aligned}
& H_{-+}\left(\bar{x} ; X_{\Omega^{\prime} c}\right) \\
& =\int_{T_{\alpha_{1}-n_{1}} \mathcal{O}_{\leq} m_{0}^{(n)}} d \lambda\left(\bar{y}^{\prime}\right) \int_{T_{\alpha_{2}+n_{1}-1} \mathcal{O}_{\leq} m_{0}^{(n)}} d \lambda\left(\bar{y}^{\prime \prime}\right) \exp \left[-h\left(\bar{y}^{\prime}\right)-h_{n}\left(T_{\alpha_{1}-n_{1}+1} \bar{x}_{-} \mid \bar{y}^{\prime}\right)\right] \\
& \quad \cdot \exp \left[-h\left(\bar{y}^{\prime \prime}\right)-h_{n}\left(T_{\alpha_{2}+n_{1}-1} \bar{x}_{+} \mid \bar{y}^{\prime \prime}\right)\right] E_{n}\left(T_{\alpha_{1}-n_{1}+1} \bar{x}_{-} \cup \bar{y}^{\prime} \mid X_{\left(-\infty, \alpha_{1}-n_{1}\right.}\right) \\
& \quad \cdot E_{n}\left(T_{\alpha_{2}+n_{1}-1} \bar{x}_{+} \cup \bar{y}^{\prime \prime} \mid X_{\left[\alpha_{2}+n_{1}, \infty\right)}\right),
\end{aligned}
$$


and

$$
\begin{aligned}
H\left(\bar{x} ; A^{-+}(\Omega)\right)= & \int_{A^{-+}(\Omega)} d \lambda(\bar{y}) \exp \left[-h\left(\bar{x}_{-}\right)-h\left(\bar{x}_{+}\right)-h(\bar{y})\right] \\
& \cdot \exp \left[-h_{n}\left(T_{\alpha_{1}-r_{n}} \bar{x}_{-} \cup T_{\alpha_{2}} \bar{x}_{+} \mid \bar{y}\right)\right] .
\end{aligned}
$$

Clearly, $H_{-+}\left(\cdot ; X_{\Omega^{\prime}}\right), H\left(\cdot ; A^{-+}(\Omega)\right) \in \mathscr{L}_{n}^{2}$.

Lemma 3.1. The following representation holds:

$$
P_{n}^{(2)}\left(A ; X_{\Omega^{\prime} c}\right)=\frac{\left\langle K_{n}^{s_{n}} H\left(\cdot ; A^{-+}(\Omega)\right), H_{-+}\left(\cdot ; X_{\Omega^{\prime} c}\right)\right\rangle}{\left\langle K_{n}^{s_{n}} H\left(\cdot ; \mathscr{Q}^{-+}(\Omega)\right), H_{-+}\left(\cdot ; X_{\Omega^{\prime} c}\right)\right\rangle} .
$$

The proof of Lemma 3.1 is immediate and based on the invariance of functions $f$ and $f_{n}$, on Lemma 1.1 and the definitions of $h_{n}$ and $P_{n}^{(2)}\left(A ; X_{\Omega^{\prime} c}\right)$.

Lemma 3.2. The operator $K_{n}$ (resp., the conjugate operator $K_{n}^{*}$ ) has a unique positive eigenvector $\Phi_{n} \in \mathscr{L}_{n}^{2}$ (resp., $\Phi_{n}^{*} \in \mathscr{L}_{n}^{2}$ ). The corresponding eigenvalue $\Lambda_{n}$ is positive, non-degenerate and the largest one among the eigenvalues of $K_{n}$ (resp., $K_{n}^{*}$ ) in $\mathscr{L}_{n}^{2}$.

Proof of Lemma 3.2. According to Proposition $\beta^{\prime}$ in [21, p. 274], it is sufficient to verify that the kernel $\mathscr{K}_{n}(\bar{x}, \bar{y})$ is strictly positive. This follows from the definition of $\mathscr{K}_{n}(\bar{x}, \bar{y})$.

In what follows we assume that the vectors $\Phi_{n}$ and $\Phi_{n}^{*}$ are chosen so that $\left\langle\Phi_{n}, \Phi_{n}^{*}\right\rangle=1$. Denote

$$
\begin{aligned}
\hat{K}_{n} & =\Lambda_{n}^{-1} K_{n}, \\
\hat{H}_{-+}\left(\bar{x} ; X_{\Omega^{\prime} c}\right) & =\left\langle H_{-+}\left(\cdot ; X_{\Omega^{\prime} c}\right), \Phi_{n}\right\rangle^{-1} H_{-+}\left(x ; X_{\Omega^{\prime} c}\right),
\end{aligned}
$$

and

$$
\hat{H}\left(\bar{x} ; A^{-+}(\Omega)\right)=\left\langle H\left(\cdot ; A^{-+}(\Omega)\right), \Phi_{n}^{*}\right\rangle^{-1} H\left(\bar{x} ; A^{-+}(\Omega)\right) \quad \text { if } \quad \lambda\left(A^{-+}(\Omega)\right)>0 ;
$$

the above formula for $P_{n}^{(2)}\left(A ; X_{\Omega^{\prime}}\right)$ may be rewritten in the form

$$
\begin{aligned}
& P_{n}^{(2)}\left(A ; X_{\Omega^{\prime} c}\right) \\
& =\left\{\begin{array}{l}
\bar{P}_{n}^{(3)}(A) \frac{1+\left\langle\hat{K}_{n}^{s_{n}} \hat{H}\left(\cdot ; A^{-+}(\Omega)\right)-\Phi_{n}, \hat{H}_{-+}\left(\cdot ; X_{\Omega^{\prime}}\right)\right\rangle}{1+\left\langle\hat{K}_{n}^{s_{n}} \hat{H}\left(\cdot ; \mathscr{Q}^{-+}(\Omega)\right)-\Phi_{n}, \hat{H}_{-+}\left(\cdot ; X_{\Omega^{\prime}}\right)\right\rangle}, \lambda\left(A^{-+}(\Omega)\right)>0, \\
0 \quad \text { otherwise, }
\end{array}\right.
\end{aligned}
$$

where

$$
\bar{P}_{n}^{(3)}(A)=\frac{\left\langle H\left(\cdot ; A^{-+}(\Omega)\right), \Phi_{n}^{*}\right\rangle}{\left\langle H\left(\cdot ; \mathscr{Q}^{-+}(\Omega)\right), \Phi_{n}^{*}\right\rangle} .
$$

Our aim now is to estimate $\left\langle\hat{K}_{n}^{s_{n}} \hat{H}\left(\cdot ; A^{-+}(\Omega)\right)-\Phi_{n}, \hat{H}_{-+}\left(\cdot ; X_{\Omega^{\prime} c}\right)\right\rangle$. To simplify the notations we omit, where it is possible, the symbols $A^{-+}(\Omega)$ and $\Omega^{\prime c}$ up to the end of the section.

Lemma 3.3. Let $X \in T_{\alpha_{1}-n_{1}-1} \mathscr{Q}_{-}^{(n)} \cap T_{\alpha_{2}+n_{1}} \mathscr{Q}_{+}^{(n)}$. Then

$$
H_{-+}(\bar{x} ; X) \leqq \exp \left[2 m_{0}^{(n)} \sum_{k \geqq 1} \psi(k) \sum_{1 \leqq j<k} m_{j}^{(n)}+2 e^{-c_{0}}\left(r_{n}+1\right)\right] \Phi_{n}^{*}(\bar{x}), \bar{x} \in \mathcal{O}^{(n)}\left(L_{n}\right) \text {. }
$$


Proof of Lemma 3.3. According to the definition we have the following estimates

$$
\begin{aligned}
& H_{-+}(\bar{x} ; X) \leqq \int_{T_{\alpha_{1}-n_{1}} \mathcal{O} \leq m_{0}^{(n)}} d \lambda\left(\bar{y}^{\prime}\right) \int_{T_{\alpha_{2}+n_{1}-1} \mathscr{C}_{\leq} m_{0}^{(n)}} d \lambda\left(\bar{y}^{\prime \prime}\right) \exp \left[-h\left(\bar{y}^{\prime}\right)-h\left(\bar{y}^{\prime \prime}\right)\right] \\
& \leqq \mu\left(\mathcal{O}_{m_{0}^{(n)}}\right)^{2} \leqq \exp \left(2 e^{-c_{0}}\right) \text {, }
\end{aligned}
$$

and

$$
\begin{aligned}
H_{-+}(\bar{x} ; X) & \geqq E_{n}\left(T_{-\alpha_{1}-n_{1}+1} \bar{x}_{-} \mid X_{\left(-\infty, \alpha_{1}-n_{1}\right.}\right) E_{n}\left(T_{\alpha_{2}+n_{1}-1} \bar{x}_{+} \mid X_{\left[\alpha_{2}+n_{1}, \infty\right)}\right) \\
& \geqq \exp \left[-2 m_{0}^{(n)} \sum_{k \geqq 1} \psi(k) \sum_{1 \leqq j \leqq k} m_{j}^{(n)}\right] .
\end{aligned}
$$

Hence,

$$
\begin{aligned}
\hat{H}_{-+}(\bar{x} ; X) \Phi_{n}^{*}(\bar{x})^{-1} \leqq & \exp \left[2 m_{0}^{(n)} \sum_{k \geqq 1} \psi(k) \sum_{1 \leqq j \leqq k} m_{j}^{(n)}+2 e^{-c_{0}}\right] \\
& \cdot\left[\Phi_{n}^{*}(\bar{x}) \int_{\mathcal{O}\left(L_{n}\right)} d \lambda(\bar{y}) \Phi_{n}(\bar{y})\right]^{-1}
\end{aligned}
$$

Now

$$
\Phi_{n}^{*}(\bar{x}) \int_{\mathscr{O}^{(n)}\left(L_{n}\right)} d \lambda(\bar{y}) \Phi_{n}(\bar{y})=\int_{\mathcal{O}^{(n)}\left(L_{n}\right)} d \lambda(\bar{y}) \Phi_{n}(\bar{y}) \Phi_{n}^{*}(\bar{y})\left[\Phi_{n}^{*}(\bar{y})^{-1} \Phi_{n}^{*}(\bar{x})\right] .
$$

The ratio $\Phi_{n}^{*}(\bar{y})^{-1} \Phi_{n}^{*}(\bar{x})$ is estimated as follows. Firstly,

$$
\begin{aligned}
& \Phi_{n}^{*}(\vec{y})^{-1} \Phi_{n}^{*}(\vec{x})=\left[\left(K_{n}^{* 2} \Phi_{n}^{*}\right)(\bar{y})\right]^{-1}\left(K_{n}^{* 2} \Phi_{n}^{*}\right)(\bar{x})
\end{aligned}
$$

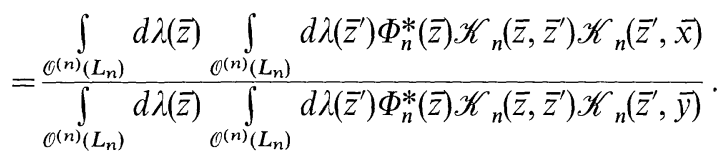

Using (*) we conclude that the denominator is less than

$$
\begin{aligned}
& \int_{\mathcal{O}(n)\left(L_{n}\right)} d \lambda(\bar{z}) \Phi_{n}^{*}(\bar{z}) \exp \left[-h\left(\bar{z}_{-}\right)-h\left(\bar{z}_{+}\right)\right] \cdot \mu\left(\mathcal{O}^{(n)}\left(L_{n}\right)\right) \\
& \leqq \int_{\mathcal{O}(n)\left(L_{n}\right)} d \lambda(\bar{z}) \Phi_{n}^{*}(\bar{z}) \exp \left[-h\left(\bar{z}_{-}\right)-h\left(\bar{z}_{+}\right)\right] \exp \left(2 r_{n} e^{-c_{0}}\right) .
\end{aligned}
$$

On the other hand the numerator is greater than

$$
\begin{aligned}
& \int_{\mathcal{O}(n)\left(L_{n}\right)} d \lambda(\bar{z}) \Phi_{n}^{*}(\bar{z}) \mathscr{K}_{n}(\bar{z}, \emptyset) \mathscr{K}_{n}(\emptyset, \bar{x}) \\
= & \int_{\mathcal{O}^{(n)}\left(L_{n}\right)} d \lambda(\bar{z}) \Phi_{n}^{*}(\bar{z}) \exp \left[-h\left(\bar{z}_{-}\right)-h\left(\bar{z}_{+}\right)\right] .
\end{aligned}
$$

Hence,

$$
\Phi_{n}^{*}(\bar{y})^{-1} \Phi_{n}^{*}(\bar{x}) \geqq \exp \left(-2 e^{-c_{0}} r_{n}\right), \bar{x}, \bar{y} \in \mathcal{O}^{(n)}\left(L_{n}\right),
$$

and, since $\left\langle\Phi_{n}, \Phi_{n}^{*}\right\rangle=1$,

$$
\Phi_{n}^{*}(\bar{x}) \int_{\mathcal{O}(n)\left(L_{n}\right)} d \lambda(\bar{y}) \Phi_{n}(\bar{y}) \geqq \exp \left(-2 e^{-c_{0}} r_{n}\right), \bar{x} \in \mathcal{O}^{(n)}\left(L_{n}\right) .
$$

This completes the proof of Lemma 3.3. 
Lemma 3.3 allows us to estimate

$$
\begin{aligned}
& \left\langle\hat{K}_{n}^{s_{n}} \hat{H}-\Phi_{n}, \hat{H}_{-+}(\cdot ; X)\right\rangle \mid \leqq\left\langle\left|\hat{K}_{n}^{s_{n}} \hat{H}-\Phi_{n}\right|, \Phi_{n}^{*}\right\rangle \\
& \cdot \exp \left[2 m_{0}^{(n)} \sum_{k \geqq 1} \psi(k) \sum_{1 \leqq j \leqq k} m_{j}^{(n)}+2 e^{-c_{0}}\left(r_{n}+1\right)\right] .
\end{aligned}
$$

The main step in the estimation of $\left\langle\hat{K}_{n}^{s_{n}} \hat{H}-\Phi_{n}, \hat{H}_{-+}(\cdot ; X)\right\rangle$ is the following.

Lemma 3.4. For any $A \in \mathfrak{B}_{\Omega}$ such that $\lambda\left(A^{-+}(\Omega)\right)>0$

$$
\left\langle\left|\hat{K}_{n}^{s_{n}} \hat{H}\left(\cdot ; A^{-+}(\Omega)\right)-\Phi_{n}\right|, \Phi_{n}^{*}\right\rangle \leqq 2\left[1-\exp \left(-4 e^{-c_{0}} r_{n}\right)\right]^{s_{n}} .
$$

Proof of Lemma 3.4 (see $[15,22]$ ). Denote

$$
\mathcal{O}_{+}^{(n)}\left(L_{n}\right)=\left\{\bar{x} \in \mathcal{O}^{(n)}\left(L_{n}\right):\left(\hat{K}_{n}^{s_{n}} \hat{H}\right)(\bar{x}) \geqq \Phi_{n}(\bar{x})\right\},
$$

and

$$
\mathcal{O}_{-}^{(n)}\left(L_{n}\right)=\left\{\bar{x} \in \mathcal{O}^{(n)}\left(L_{n}\right):\left(\hat{K}_{n}^{s_{n}} \hat{H}\right)(\bar{x}) \leqq \Phi_{n}(\bar{x})\right\} ;
$$

the both sets depend, of course, on $A^{-+}(\Omega)$. Since $\left\langle\hat{K}_{n}^{s_{n}} \hat{H}-\Phi_{n}, \Phi_{n}^{*}\right\rangle$ $=\left\langle\hat{H}, \hat{K}_{n}^{* s_{n}} \Phi_{n}^{*}\right\rangle-1=\left\langle\hat{H}, \Phi_{n}^{*}\right\rangle-1=0$, we obtain

$$
\begin{aligned}
& \left\langle\mid \hat{K}_{n}^{s_{n}} \hat{H}-\Phi_{n}, \Phi_{n}^{*}\right\rangle=2 \int_{\mathscr{O}_{+}^{(n)}\left(L_{n}\right)} d \lambda(\bar{x})\left[\left(\hat{K}_{n}^{s_{n}} \hat{H}\right)(\bar{x})-\Phi_{n}(\bar{x})\right] \Phi_{n}^{*}(\bar{x}) \\
& =2 \int_{\left.\mathscr{O}^{(n)}\right)\left(L_{n}\right)} d \lambda(\bar{x}) \Phi_{n}^{*}(\bar{x}) \int_{\mathscr{O}^{(n)}\left(L_{n}\right)} d \lambda(\bar{y}) \Lambda_{n}^{-1} \mathscr{K}_{n}(\bar{x}, \bar{y})\left[\left(\hat{K}_{n}^{s_{n}-1} \hat{H}\right)(\bar{y})-\Phi_{n}(\bar{y})\right] \\
& =2 \int_{\left({ }^{(n)}\right)\left(L_{n}\right)} d \lambda(\vec{y})\left[\left(K_{n}^{s_{n}-1} \hat{H}\right)(\bar{y})-\Phi_{n}(\bar{y})\right] \Phi_{n}^{*}(\bar{y}) \\
& \cdot \int_{\mathscr{O}_{+}^{(n)}(L n)} d \lambda(\bar{x}) \Phi_{n}^{*}(\bar{x})\left[\Lambda_{n}^{-1} \mathscr{K}_{n}(\bar{x}, \bar{y})\right] \Phi_{n}^{*}(\bar{y})^{-1} \\
& =2 \int_{\mathscr{O}(n)\left(L_{n}\right)} d \lambda(\bar{y})\left[\left(\hat{K}_{n}^{s_{n}-1} \hat{H}\right)(\vec{y})-\Phi_{n}(\vec{y})\right] \Phi_{n}^{*}(\vec{y}) \\
& \cdot\left(1-\int_{\mathscr{O}(n)\left(L_{n}\right)} d \lambda(\bar{x}) \Phi_{n}^{*}(\bar{x})\left[\Lambda_{n}^{-1} \mathscr{K}_{n}(\bar{x}, \bar{y})\right] \Phi_{n}^{*}(\bar{y})^{-1}\right) .
\end{aligned}
$$

Now denote

$$
\begin{aligned}
& \mathcal{O}_{++}^{(n)}\left(L_{n}\right)=\left\{\bar{x} \in \mathcal{O}^{(n)}\left(L_{n}\right):\left(\hat{K}_{n}^{s_{n}-1} \hat{H}\right)(\bar{x}) \geqq \Phi_{n}(\bar{x})\right\}, \\
& \mathcal{O}_{-}^{(n)}\left(L_{n}\right)=\left\{\bar{x} \in \mathcal{O}^{(n)}\left(L_{n}\right):\left(K_{n}^{s_{n}-1} \hat{H}\right)(\bar{x})<\Phi_{n}(\bar{x})\right\} .
\end{aligned}
$$

Then the RHS of the last equality is less or equal than

$$
\begin{aligned}
& 2 \int_{\mathcal{O}^{(n)}\left(L_{n}\right)} d \lambda(\bar{y})\left[\left(\hat{K}_{n}^{s_{n}-1} \hat{H}\right)(\bar{y})-\Phi_{n}(\bar{y})\right] \Phi_{n}^{*}(y) \\
& \cdot\left(1-\int_{\mathscr{O}(n)\left(L_{n}\right)} d \lambda(\bar{x}) \Phi_{n}^{*}(\bar{x})\left[\Lambda_{n}^{-1} \mathscr{K}_{n}(\bar{x}, \bar{y})\right] \Phi_{n}^{*}(\bar{y})^{-1}\right) .
\end{aligned}
$$

There are two possibilities: $\emptyset \in \mathcal{O}_{-}^{(n)}\left(L_{n}\right)$ and $\emptyset \in \mathcal{O}_{+}^{(n)}\left(L_{n}\right)$. At first suppose that $\emptyset \in \mathcal{O}_{-}^{(n)}\left(L_{n}\right)$. Then according to the above estimate for $\Phi_{n}^{*}(\bar{y})^{-1} \Phi_{n}(\bar{x})$,

$$
\begin{aligned}
& \int_{\mathscr{O}\left(\underline{n)}\left(\Lambda_{n}\right)\right.} d \lambda(\bar{x}) \Phi_{n}^{*}(\bar{x})\left[\Lambda_{n}^{-1} \mathscr{K}_{n}(\bar{x}, \bar{y})\right] \Phi_{n}^{*}(\bar{y})^{-1} \\
& \geqq \Phi_{n}^{*}(\emptyset)\left[\Lambda_{n}^{-1} \mathscr{K}_{n}(\emptyset, \bar{y})\right] \Phi_{n}^{*}(\bar{y})^{-1} \geqq \Lambda_{n}^{-1} \exp \left(-2 e^{-c_{0}} r_{n}\right) .
\end{aligned}
$$

According to Lemma 3.2, for any fixed positive $F_{1}, F_{2} \in \mathscr{L}_{n}^{2}$

$$
\lim _{k \rightarrow \infty}\left\langle K_{n}^{k} F_{1}, F_{2}\right\rangle=\Lambda_{n} \text {. }
$$


Since $\mathscr{K}_{n}(\bar{x}, \bar{y}) \leqq \exp \left[-h\left(\bar{x}_{-}\right)-h\left(\bar{x}_{+}\right)\right]$, we deduce from this that $\Lambda_{n} \leqq \mu\left(\mathcal{O}^{(n)}\left(\Lambda_{n}\right)\right)<$ $\exp \left(2 e^{-c_{0}} r_{n}\right)$, and hence the RHS of the preceding estimate is greater or equal to $\exp \left(-4 e^{-{ }^{c_{0}}} r_{n}\right)$. Finally, in the case $\emptyset \in \mathcal{O}_{-}^{(n)}\left(L_{n}\right)$ we obtain

$$
\begin{aligned}
& \left\langle\left|\hat{K}_{n}^{s_{n}} \hat{H}-\Phi_{n}\right|, \Phi_{n}^{*}\right\rangle \leqq 2\left[1-\exp \left(-4 e^{-c_{0}} r_{n}\right)\right]
\end{aligned}
$$

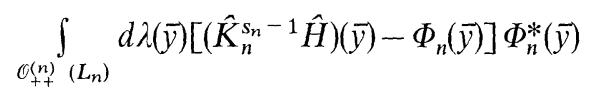

$$
\begin{aligned}
& =\left[1-\exp \left(-4 e^{-c_{0}} r_{n}\right)\right]\left\langle\left|\hat{K}_{n}^{s_{n}-1} \hat{H}-\Phi_{n}\right|, \Phi_{n}^{*}\right\rangle ;
\end{aligned}
$$

the last equality holds because $\left\langle\hat{K}_{n}^{s_{n}-1} \hat{H}-\Phi_{n}, \Phi_{n}^{*}\right\rangle=0$.

If $\emptyset \in \mathcal{O}_{+}^{(n)}\left(L_{n}\right)$, we can repeat the arguments replacing everywhere $\mathcal{O}_{+}^{(n)}\left(L_{n}\right)$ and $\mathcal{O}_{++}^{(n)}\left(L_{n}\right)$ by $\mathcal{O}_{-}^{(n)}\left(L_{n}\right)$ and $\mathcal{O}_{--}^{(n)}\left(L_{n}\right)$ and vice versa. We arrive to the same bound.

By repeating this procedure $s_{n}$ times and taking into account that $\left\langle\left|\hat{H}-\Phi_{n}\right|\right.$, $\left.\Phi_{n}^{*}\right\rangle \leqq\left\langle\hat{H}+\Phi_{n}, \Phi_{n}^{*}\right\rangle=2$, we obtain the statement of Lemma 3.4. Q.E.D.

According to Lemmas 3.3 and 3.4, we obtain the estimate

$$
\begin{aligned}
& \left\langle\hat{K}^{s_{n}} \hat{H}\left(\cdot ; A^{-+}(\Omega)\right)-\Phi_{n}, \hat{H}_{-+}\left(\cdot ; X_{\Omega^{\prime} c}\right)\right\rangle \mid \\
& \leqq 2 \exp \left[2 m_{0}^{(n)} \sum_{k \geqq 1} \psi(k) \sum_{1 \leqq j \leqq k} m_{j}^{(n)}+2 e^{-c_{0}}\left(r_{n}+1\right)-S_{n} e^{-4 c_{0} r_{n}}\right] .
\end{aligned}
$$

With our choice of $n$ (see $(* *))$ the following lemma holds:

Lemma 3.5. Let $P$ satisfy the $D L R$ equation with the g.f. $f$. Then for any $A \in \mathfrak{B}_{\Omega}$

$$
\left|P_{n}^{(2)}(A)-\bar{P}_{n}^{(3)}(A)\right|<1 / 6 \varepsilon .
$$

Proof of Lemma 3.5. Clearly, it is sufficient to consider the case $\lambda\left(A_{\Omega}^{-+}\right)>0$. According to preceding estimates,

$$
\left|\left(P_{\Omega^{\prime}}^{\Omega}\right)_{n}^{(2)}\left(A ; X_{\Omega^{\prime} c}\right)-\bar{P}_{n}^{(3)}(A)\right|<2 / 28 \varepsilon(1-1 / 28 \varepsilon)^{-1}<1 / 7 \varepsilon .
$$

Integrating over $T_{\alpha_{1}-n_{1}-1} \mathscr{2}_{-}^{(n)} \cap T_{\alpha_{2}+n_{1}} \mathscr{2}_{+}^{(n)}$ and taking into account that $P\left(T_{\alpha_{1}-n_{1}-1} \mathscr{Q}_{-}^{(n)} \cap T_{\alpha_{2}+n_{1}} \mathscr{Q}_{+}^{(n)}\right) \geqq 1-1 / 42 \varepsilon$, we obtain the statement of Lemma 3.5 .

Now suppose there are two solutions, $P^{\prime}$ and $P^{\prime \prime}$, of the DLR equation with the g.f. $f$. Then, according to Lemmas $2.2,2.3$ and 3.5 , for any $A \in \mathfrak{B}_{\Omega}$,

$$
\left|P^{\prime}(A)-\bar{P}_{n}^{(3)}(A)\right|<1 / 2 \varepsilon,\left|P^{\prime \prime}(A)-\bar{P}_{n}^{(3)}(A)\right|<1 / 2 \varepsilon,
$$

i.e., $\left|P^{\prime}(A)-P^{\prime \prime}(A)\right|<\varepsilon$. Since $\varepsilon$ is arbitrary small, $P^{\prime}(A)=P^{\prime \prime}(A)$. This completes the proof of Theorem 1.

We conclude this section by a remark used below. The arguments of Sections 2, 3 may be summarized as

Lemma 3.6. Let $P$ be a solution of the DLR equation with the g.f.f. Given $\varepsilon>0$ there exists $n$ such that for any $\Omega=\left[\alpha_{1}, \alpha_{2}\right)$ where $\alpha_{1}<\alpha_{2}$ are integers, any $A \in \mathfrak{B}_{\Omega}$ and $X \in T_{\alpha_{1}-n_{1}-1} \mathscr{Q}_{-}^{(n)} \cap T_{\alpha_{2}+n_{1}} \mathscr{Q}_{+}^{(n)}$

$$
\left|P_{\Omega^{\prime}}^{\Omega}\left(A ; X_{\Omega^{\prime} c}\right)-P(A)\right|<\varepsilon .
$$

We emphasize that although all constructions of the two last sections are given for fixed $\Omega$, the choice of $n$ does not depend on it. This fact will be used in the proof of Theorems 2 and 3 . 


\section{Conditional DLR Equation}

Ergodic properties of the r.p.p. $P$ being the solution of the DLR equation with the g.f. $f$ are closely connected with the conditional probability measure $P\left(\cdot \mid \mathfrak{B}_{[0, \infty)}\right)$ where the $\sigma$-algebra $\mathfrak{B}_{[0, \infty)}$ is the smallest one containing all $\mathfrak{B}_{\Omega}$, $\Omega \subset[0, \infty)$. A useful construction relevant to $P\left(\cdot \mid \mathfrak{B}_{[0, \infty)}\right)$ may be given as follows.

Denote

$$
\hat{\mathscr{Q}}=\mathscr{Q} \backslash \bigcap_{k} \bigcup_{n \geqq k} \overline{\mathscr{Q}}_{+}^{(n)}
$$

In other terms, $X \in \hat{\mathscr{Q}}$ iff for almost all $n, X \in \mathscr{Q}_{-}^{(n)}$. Clearly, $\hat{\mathscr{Q}} \in \mathfrak{B}$, and $P(\mathscr{Q} \backslash \hat{\mathscr{Q}}) \leqq$ inf $\sum_{n \geqq k} P\left(\overline{\mathscr{Q}}_{+}^{(n)}\right)=0$ according to Lemma 2.1, i.e., $P(\hat{\mathscr{Q}})=1$.

Let $X \in \mathscr{Q}$; we say that a probability measure $\mathscr{P}_{(-\infty, 0)}(\cdot ; X)$ on the $\sigma$-algebra $\mathfrak{B}_{(-\infty, 0)}$ generated by $\mathfrak{B}_{\Omega}, \Omega \subset(-\infty, 0)$, is a solution of the conditional DLR equation with the g.f. $f$ if for any b.B.s. $\Omega \subset(-\infty, 0)$ and $A \in \mathfrak{B}_{\Omega}$

$$
\mathscr{P}_{(-\infty, 0)}(A ; X)=\int_{\mathscr{Q}} \mathscr{P}_{(-\infty, 0)}(d Y ; X) P_{\Omega}\left(A ; Y_{(-\infty, 0) \backslash \Omega} \cup X_{[0, \infty)}\right) \text {. }
$$

Theorem 1'. Let $f$ satisfy the conditions (I1-I3). For any $X \in \hat{\mathscr{Z}}$ there exists a unique solution $\mathscr{P}_{(-\infty, 0)}(\cdot ; X)$ of the conditional $D L R$ equation with the g.f. $f$. If $P$ is the solution of the DLR equation with the same g.f., then

$$
\mathscr{P}_{(-\infty, 0)}(\cdot ; X)=P\left(\cdot \mid \mathfrak{B}_{[0, \infty)}\right)(X)
$$

for P-almost all $X$.

Proof of Theorem 1'. We give here a sketch of the proof omitting details which are analogous to that in the proof of Theorem 1 . The existence of a solution for $X \in \hat{\mathcal{Q}}$ may be proved, as above, by using the methods of [8]. So we prove the uniqueness. Given $X \in \hat{\mathscr{Q}}$, there exists $n_{0}=n_{0}(X)$ such that $X \in \mathscr{Q}_{+}^{(n)}$ for any $n \geqq n_{0}$. Given $\varepsilon>0$, choose $n$ so large that $n \geqq n_{0}$ and the inequalities (**) hold. Let $\Omega=\left[\alpha_{1}, 0\right)$ where $\alpha_{1}<0$ is an integer; the notations $n_{1}$ and $\Omega^{-}$used below have the same sense as in Sections 2, 3. Denote $\Omega_{1}^{-}=\Omega^{-} \cup \Omega=\left[\alpha_{1}-n_{1}, 0\right)$ and for $A \in \mathfrak{B}_{\Omega}$

\section{Denote}

$$
A^{+}\left(\alpha_{1}\right)=\left(\pi_{\Omega} A\right) \cap\left(\bigcap_{k=0}^{-\alpha_{1}-1} T_{\alpha_{1}+k} \mathcal{O}_{\leqq m_{k+1}^{(n)}}\right) .
$$

$$
\begin{aligned}
& \tilde{\Xi}_{n}^{(1)}\left(\Omega_{1}^{-} ; Y_{\left(-\infty, \alpha_{1}-n_{1}\right)} \cup X_{[0, \infty)}\right) \\
& =\int_{2^{+}\left(\alpha_{1}\right)} d \lambda(\bar{y}) \int_{\mathcal{O}^{(n)}\left(\Omega^{-}\right)} d \lambda\left(\bar{y}^{-}\right) \exp \left[-h\left(\bar{y} \cup \bar{y}^{-}\right)\right] E\left(\bar{y} \cup \bar{y}^{-} \mid Y_{\left(-\infty, \alpha_{1}-n_{1}\right)} \cup X_{[0, \infty)}\right), \\
& \tilde{\Xi}_{\Omega, n}^{(1)}\left(\bar{x}, \Omega_{1}^{-} ; Y_{\left(-\infty, \alpha_{1}-n_{1}\right)} \cup X_{[0, \infty)}\right) \\
& =\int_{\mathcal{O}^{(n)}\left(\Omega^{-}\right)} d \lambda\left(\bar{y}^{-}\right) \exp [-h(\bar{x} \cup \bar{y})] E\left(\bar{x} \cup \bar{y} \mid Y_{\left(-\infty, \alpha_{1}-n_{1}\right)} \cup X_{[0, \infty}\right), \\
& \left(\tilde{p}_{\Omega_{1}^{-}}^{\Omega(1)}\right)_{n}^{(1)}\left(\bar{x} ; Y_{\left(-\infty, \alpha_{1}-n_{1}\right)} \cup X_{[0, \infty)}\right) \\
& =\tilde{\Xi}_{n}^{(1)}\left(\Omega_{1}^{-} ; Y_{\left(-\infty, \alpha_{1}-n_{1}\right)} \cup X_{[0, \infty)}\right)^{-1} \tilde{\Xi}_{\Omega, n}^{(1)}\left(\bar{x}, \Omega_{1}^{-} ; Y_{\left(-\infty, \alpha_{1}-n_{1}\right)} \cup X_{[0, \infty)}\right) .
\end{aligned}
$$

To simplify the notations we omit, where it is possible, the upper and lower indices $\Omega, \Omega_{1}^{-},(-\infty, 0)$, etc., keeping in mind that $\tilde{p}_{n}^{(i)}(\bar{x} ; Y, X)$ below denotes 
$\left(\tilde{p}_{\Omega_{1}}^{\Omega}\right)_{n}^{(i)}\left(\bar{x} ; Y_{\left(-\infty, \alpha_{1}-n_{1}\right)} \cup X_{[0, \infty)}\right), i=1,2$, etc. We first approximate $\mathscr{P}(A ; X)$ by

$$
\mathscr{P}_{n}^{(1)}(A ; X)=\int_{A^{+}\left(\alpha_{1}\right)} d \lambda(\bar{x}) \int_{T_{\alpha_{1}-n_{1}-1} \mathscr{Q}_{(n)}^{(n)}} \mathscr{P}(d Y ; X) \tilde{p}_{n}^{(1)}(\bar{x} ; Y, X) \text {. }
$$

Lemma 4.1. Let $\mathscr{P}(\cdot ; X)$ be a solution of the conditional DLR equation with the g.f. $f$ obeying (I1-I3). Then for any $A \in \mathfrak{B}_{\Omega}$

$\left|\mathscr{P}(A ; X)-\mathscr{P}_{n}^{(1)}(A ; X)\right|<1 / 6 \varepsilon$.

The proof of Lemma 4.1 is analogous to that of Lemma 2.2 and we omit it. The next approximation is

$$
\begin{aligned}
& \tilde{\Xi}_{n}^{(2)}\left(\Omega_{1}^{-} ; Y, X\right) \\
& =\int_{2^{+}\left(\alpha_{1}\right)} d \lambda(\bar{y}) \int_{\mathscr{O}^{(n)}\left(\Omega^{-}\right)} d \lambda\left(\bar{y}^{-}\right) \exp \left[-h(\bar{y})-h_{n}\left(\bar{y}^{-}\right)-h_{n}\left(\bar{y} \mid \bar{y}^{-}\right)\right] E_{n}\left(\bar{y} \cup \bar{y}^{-} \mid Y, X\right), \\
& \tilde{\Xi}_{\Omega, n}^{(2)}\left(\bar{x}, \Omega_{1}^{-} ; Y, X\right) \\
& =\int_{\mathcal{O}^{(n)}\left(\Omega^{-}\right)} d \lambda\left(\bar{y}^{-}\right) \exp \left[-h(\bar{x})-h_{n}\left(\bar{y}^{-}\right)-h_{n}\left(\bar{x} \mid \bar{y}^{-}\right)\right] E_{n}\left(\bar{x} \cup \bar{y}^{-} \mid Y, X\right), \\
& \tilde{p}_{n}^{(2)}(\bar{x} ; X, Y)=\tilde{\Xi}_{n}^{(2)}\left(\Omega_{1}^{-} ; X, Y\right)^{-1} \tilde{\Xi}_{n}^{(2)}\left(\bar{x}, \Omega_{1} ; Y, X\right),
\end{aligned}
$$

and

$$
\mathscr{P}_{n}^{(2)}(A ; X)=\int_{A^{+}\left(\alpha_{1}\right)} d \lambda(\bar{x}) \int_{T_{\alpha_{1}-n_{1}-1} \mathscr{2}(n)} \mathscr{P}(d Y ; X) \tilde{p}_{n}^{(2)}(\bar{x} ; Y, X) .
$$

Lemma 4.2. Let $\mathscr{P}(\cdot ; X)$ be a solution of the conditional DLR equation with the g.f. $f$ where $f$ satisfies the conditions (I1-I3). Then for any $A \in \mathfrak{B}_{\Omega}$

$$
\left|\mathscr{P}_{n}^{(1)}(A ; X)-\mathscr{P}_{n}^{(2)}(A ; X)\right|<1 / 6 \varepsilon .
$$

The proof of Lemma 4.2 is analogous to that of Lemma 2.3 and omitted. Denote $L_{n}^{-}=\left[-r_{n}, 0\right)$ and $\mathcal{O}^{(n)}\left(L_{n}^{-}\right)=\mathcal{O}\left(L_{n}\right) \cap\left(\bigcap_{k=-r_{n}}^{-1} T_{k} \mathcal{O}_{\leqq}\right) ;$in $)$; in section $\mathscr{L}_{n}^{2}$ denotes the space $\mathscr{L}^{2}\left(\mathcal{O}^{(n)}\left(L_{n}^{-}\right), \lambda\right)$ and $\langle$,$\rangle its inner product. Let$

$$
\mathscr{K}_{n}^{-}(\bar{x}, \bar{y})=\exp \left[-h(\bar{x})-h_{n}\left(T_{-r_{n}} \bar{x} \mid \bar{y}\right)\right], \bar{x}, \bar{y} \in \mathcal{O}^{(n)}\left(L_{n}^{-}\right),
$$

consider the operator $K_{n}^{-}$in $\mathscr{L}_{n}^{2}$ given by

$$
\left(K_{n}^{-} F\right)(\bar{x})=\int_{\mathcal{O}^{(n)}\left(L_{n}\right)} d \lambda(\bar{y}) \mathscr{K}_{n}^{-}(\bar{x}, \vec{y}) F(\vec{y}) .
$$

Introducing the functions

and

$$
\begin{aligned}
H_{-}(\bar{x})= & \int_{T_{\alpha_{1}-n_{1} O \leq m_{0}^{(n)}}} d \lambda\left(\bar{y}^{\prime}\right) \int_{T_{\alpha_{1}-n_{1}-1} \mathscr{L}_{-}^{(n)}} \mathscr{P}(d Y ; X) \exp \left[-h\left(\bar{y}^{\prime}\right)\right. \\
& \left.-h_{n}\left(T_{\alpha_{1}-n_{1}+1} \bar{x} \mid \bar{y}^{\prime}\right)\right], \\
& E_{n}\left(T_{\alpha_{1}-n_{1}+1} \bar{x} \cup \bar{y}^{\prime} \mid Y_{\left(-\infty, \alpha_{1}-n_{1}\right)}\right), \bar{x} \in \mathcal{O}^{(n)}\left(L_{n}^{-}\right),
\end{aligned}
$$

$$
\begin{gathered}
H\left(\bar{x}, A^{+}\left(\alpha_{1}\right) ; X\right)=\int_{A^{+}\left(\alpha_{1}\right)} d \lambda(\bar{y}) \exp \left[-h(\bar{y})-h_{n}\left(T_{\alpha_{1}-r_{n}} \bar{x} \mid \bar{y}\right)\right] \\
E_{n}\left(T_{\alpha_{1}-r_{n}} \bar{x} \cup \bar{y} \mid X_{[0, \infty)}\right), \bar{x} \in \mathcal{O}^{(n)}\left(L_{n}^{-}\right),
\end{gathered}
$$

we have the following 


\section{Lemma 4.3.}

$$
\mathscr{P}_{n}^{(2)}(A ; X)=\frac{\left\langle\left(K_{n}^{-}\right)^{s_{n}} H\left(\cdot ; A^{+}\left(\alpha_{1}\right) ; X\right), H_{-}\right\rangle}{\left\langle\left(K_{n}^{-}\right)^{s_{n}} H\left(\cdot, \mathscr{Q}^{+}\left(\alpha_{1}\right) ; X\right), H_{-}\right\rangle} .
$$

The spectral analysis of the operator $K_{n}^{-}$is analogous to that of $K_{n}$. Finally, introducing

$$
\overline{\mathscr{P}}_{n}^{(3)}(A)=\frac{\left\langle H\left(\cdot ; A^{+}\left(\alpha_{1}\right), X\right), \Phi_{n}^{-}, *\right\rangle}{\left\langle H\left(\cdot ; \mathscr{Q}^{+}\left(\alpha_{1}\right), X\right), \Phi_{n}^{-, *}\right\rangle}
$$

where $\Phi_{n}^{-}$,* is the positive eigenvector of the conjugate operator $\left(K_{n}^{-}\right)^{*}$, we obtain an assertion which is analogous to Lemma 3.5.:

Lemma 4.4. If $\mathscr{P}(\cdot ; X)$ satisfies the conditional $D L R$ equation with the g.f. $f$, then for any $A \in \mathfrak{B}_{\Omega}$

$$
\left|\mathscr{P}_{n}^{(2)}(A ; X)-\overline{\mathscr{P}}_{n}^{(3)}(A)\right|<1 / 6 \varepsilon .
$$

Now the proof of the uniqueness of the solution of the conditional DLR equation is completed as above.

The proof of the equality $\mathscr{P}_{(-\infty, 0)}(\cdot ; X)=P\left(\cdot \mid \mathfrak{B}_{[0, \infty)}\right)(X)$ is based on applications of Fubini's theorem and the proved uniqueness of the solution of the (unconditional) DLR equation. We omit it from the paper.

We summarize the above considerations as follows.

Lemma 4.5. Let $P$ (resp., $\left.\mathscr{P}_{(-\infty, 0)}(\cdot ; X), X \in \hat{\mathscr{Q}}\right)$ be the solution of the DLR (resp., conditional DLR) equation with the g.f. $f$ satisfying the conditions (I1-I3). Given $\varepsilon>0$, there exists $n$ such that for every $X \in \hat{\mathscr{Q}} \cap \mathscr{Q}_{+}^{(n)}$

$$
\sup _{n^{\prime}>n_{1}} \operatorname{Var}\left[\mathscr{P}_{(-\infty, 0)}^{\left[-n^{\prime},-n_{1}\right)}(\cdot ; X), P^{\left[-n^{\prime},-n_{1}\right)}\right]<\varepsilon,
$$

where $\mathscr{P}_{(-\infty, 0)}^{\left[-n^{\prime},-n_{1}\right)}(\cdot ; X)$ is the restriction of the probability measure $\mathscr{P}_{(-\infty, 0)}(\cdot ; X)$ on $\mathfrak{B}_{\left[-n^{\prime},-n_{1}\right)}$.

Proof of Lemma 4.5. Given $\varepsilon>0$ choose $n$ such that

a) for every $k=1,2, \ldots$, and any $X \in \hat{\mathscr{Q}} \cap \mathscr{Q}_{+}^{(n)}, \mathscr{P}\left(T_{-k^{2}} \overline{\mathscr{Q}}_{-}^{(n)} ; X\right)<1 / 3 \varepsilon$,

b) for every $\Omega=\left[\alpha_{1}, \alpha_{2}\right)$, any $X \in T_{\alpha_{1}-n_{1}-1} \mathcal{Q}_{-}^{(n)} \cap T_{\alpha_{2}+n_{1}} \mathcal{Q}_{+}^{(n)}$ and $A \in \mathfrak{B}_{\Omega}$,

$$
\left|P_{\Omega^{\prime}}^{\Omega}\left(A ; X_{\Omega^{\prime}}\right)-P(A)\right|<1 / 3 \varepsilon \text {. }
$$

The first possibility follows from the definition of $\mathscr{P}_{(-\infty, 0)}(\cdot ; X)$ and the estimates (**), while the second one is the assertion of Lemma 3.6. According to the definition of $\mathscr{P}_{(-\infty, 0)}(\cdot ; X)$, for every $n^{\prime}>n_{1}$ and any $A \in \mathfrak{B}_{\left[-n^{\prime},-n_{1}\right)}$ we have

$$
\begin{aligned}
& \left|\mathscr{P}_{(-\infty, 0)}(A ; X)-P(A)\right| \\
& \leqq \int_{T_{-n^{\prime}-n_{1}-1} \mathscr{2}(n)} \mathscr{P}_{(-\infty, 0)}(d Y ; X)\left|P_{\left[-n^{\prime}-n_{1}, 0\right)}^{\left[-n^{\prime},-n_{1}\right)}\left(A ; Y_{\left(-\infty,-n^{\prime}-n_{1}\right)} \cup X_{[0, \infty)}\right)-P(A)\right| \\
& +\int_{T-n^{\prime}-n_{1}-1^{2}(n)} \mathscr{P}_{(-\infty, 0)}(d Y ; X) P_{\left[-n^{\prime}-n_{1}, 0\right)}^{\left[-n^{\prime},-n_{1}\right)}\left(A ; Y_{\left(-\infty,-n^{\prime}-n_{1}\right)} \cup X_{[0, \infty)}\right) \\
& +\mathscr{P}_{(-\infty, 0)}\left(T_{-n^{\prime}-n_{1}} \overline{\mathscr{Q}}_{-}^{(n)}\right) P(A),
\end{aligned}
$$

and according to the conditions a), b), the RHS of the inequality is less than $\varepsilon$. Taking the suprema in the left hand side (LHS) of the inequality we arrive to the assertion of Lemma 4.5. Q.E.D. 


\section{Proof of Theorem 2}

In this short section we prove the regularity of the r.p.p. $P$. Clearly we can consider only the case $\Omega=[-\alpha, \alpha)$ where $0<\alpha<\infty$ is an integer. It suffices to find a subset $\hat{\mathscr{Q}}^{\prime} \subset \mathscr{Q}, \hat{\mathscr{Q}}^{\prime} \in \mathfrak{B}$, such that $P\left(\hat{\mathscr{Q}}^{\prime}\right)=1$, and for any $X \in \hat{\mathscr{Q}}^{\prime}$

$\lim _{N \rightarrow \infty} \operatorname{Var}\left[P_{\Omega_{N}}^{\Omega}\left(A ; X_{\Omega_{N}^{c}}\right), P^{\Omega}\right]=0$.

We choose $\quad \hat{\mathscr{Q}}^{\prime}=\mathscr{2} \backslash\left[\bigcap_{k} \bigcup_{n \geqq k}\left(T_{-\alpha-n_{1}-1} \overline{\mathscr{Q}}_{-}^{(n)} \cap T_{\alpha+n_{1}} \overline{\mathscr{Q}}_{+}^{(n)}\right)\right]\left(\right.$ remember: $n_{1}=n_{1}(n)=$ $\left.r_{n}\left(s_{n}+1\right)+1\right)$. In other terms, $X \in \hat{\mathscr{Q}}^{\prime}$ iff for almost all $n, X \in T_{-\alpha-n_{1}-1} \mathscr{Q}_{-}^{(n)} \cap T_{\alpha+n_{1}} \mathscr{Q}_{+}^{(n)}$. As above, it is not hard to see that such $\hat{\mathscr{Q}}^{\prime}$ has $P$-mesure 1 .

We now prove the limit relation above. Given $X \in \hat{\mathscr{Q}}^{\prime}$, there exists $n_{0}=n_{0}(X)$ such that $X \in T_{-\alpha-n_{1}-1} \mathcal{Q}_{-}^{(n)} \cap T_{\alpha+n_{1}} \mathcal{Q}_{+}^{(n)}$ for every $n \geqq n_{0}$. Given $\varepsilon>0$, choose $n$ so large that $n \geqq n_{0}$ and the assertion of Lemma 3.6 holds. Take $N_{0}=\alpha+n_{1}$. Then for all $N \geqq N_{0}$

a) $X \in T_{-N-1} \mathscr{Q}_{-}^{(n)} \cap T_{N} \mathscr{Q}_{+}^{(n)}$ because of the monotonicity of $m_{j}^{(n)}, j=1,2 \ldots$,

b) $P_{\Omega_{N}}^{\Omega}\left(A ; X_{\Omega_{N}^{c}}\right)=P_{\tilde{\Omega}^{\prime}}^{\tilde{\Omega}^{\prime}}\left(A ; X_{\tilde{\Omega}^{\prime} c}\right)$ where $\tilde{\Omega}=\left(-\alpha-N+N_{0}, \alpha+N-N_{0}\right)$.

Hence, according to Lemma 3.6,

$\left|P_{\Omega_{N}}^{\Omega}\left(A ; X_{\Omega_{N}^{c}}\right)-P(A)\right|<\varepsilon$.

Taking the supremum of the LHS of the last inequality we obtain the assertion of Theorem 2.

\section{Proof of Theorem 3}

The invariance of $P$ follows from Theorem 5.8 of [8] (see the footnote 6,p.119). For reader's convenience we give a sketch of a direct proof. By the invariance of the g.f. $f$, for any b.B.s. $\Omega \subset \Omega^{\prime} \subset R^{1}$

$$
P_{\Omega^{\prime}}^{\Omega}(A ; X)=P_{T_{t} \Omega^{\prime}}^{T_{t} \Omega_{t}}\left(T_{t} A ; T_{t} X\right) .
$$

Then the assertion of Lemma 3.6 implies that $\left|P(A)-P\left(T_{t} A\right)\right|<2 \varepsilon$. This gives the invariance of $P$.

In what follows we use standard notions and notations from ergodic theory (see, e.g. [20]). The assertion of Theorem 3 means that for every $t \in R^{1}, T_{t}$ is a generalized B-shift. We prove this for $t=1$, the general case may be considered in a similar way, using a slight modification of the construction of sections 2-3. The key statement in the proof of the B-property of the r.p.p. $P$ is Lemma 4.5.

Let $\xi$ be the countable partition of $\mathscr{Q}$ whose elements are

$$
C_{m}=\left\{X \in \mathscr{Q}: X \in \pi_{[0,1)}^{-1} \mathcal{O}_{m}\right\}, m=0,1, \ldots .
$$

Let $\eta_{i}$ be a decreasing sequence of $\mathfrak{B}_{[0,1)}$-mesurable countable partitions of $\mathscr{Q}$ such that $\eta_{l} \geqq \xi$ and $\bigvee_{l} \bar{\eta}_{l}=\mathfrak{B}$ where $\bar{\eta}_{l}=\bigvee_{k=-\infty}^{\infty} T_{k} \eta_{l}$. [It is easy to give examples of such sequences, see, e.g., [23].] According to Theorem 2 of [24] it is sufficient to verify that every $\eta_{i}$ is weak Bernoulli. Take $\varepsilon>0$ and choose $n$ so large that $P\left(\overline{\mathscr{Q}}_{+}^{(n)}\right) \leqq \sum_{k=0}^{\infty} P\left(T_{k^{2}} \mathscr{Q}_{m_{k+1}^{(n)}}\right) \leqq \sum_{k=1}^{\infty} \mu\left(\mathcal{O}_{>m_{k+1}^{(n)}}\right)<a_{n}^{(1)}<\varepsilon$, and the assertion of Lemma 4.5 holds. Let $n^{\prime}>n_{1}$ be an integer; denote by $\left\{D_{i}^{\prime}\right\}$ (resp., $\left\{D_{j}^{\prime \prime}\right\}$ ) the collection of 
elements of $\bigvee_{k=0}^{n^{\prime}-n_{1}} T_{k} \eta_{l}\left(\right.$ resp., $\left.\bigvee_{k=-n^{\prime}}^{-n_{1}} T_{k} \eta_{l}\right)$, and by $E^{(n)}$ the union of those $D_{i}^{\prime}$ which either belong to $\bigcup_{k=0} T_{k} \mathscr{Q}_{>m_{k+1}^{(n)}}$ or have the $P$-measure 0 . By the choice of $n$, $P\left(E^{(n)}\right)<\varepsilon$.

Now let $D_{i}^{\prime} \oplus E^{(n)}$. Then

$$
\begin{aligned}
& P\left(D_{i}^{\prime} \cap \overline{\mathscr{Q}}_{+}^{(n)}\right) \leqq \sum_{k \geqq n} P\left(D_{i}^{\prime} \cap T_{k^{2} m_{k+1}^{(n)}}\right) \\
& \leqq \sum_{k \geqq n} P\left(D_{i}^{\prime} \cap \pi_{[k, k+1)}^{-1} \emptyset\right) \cdot \mu\left(\mathcal{O}_{m_{k}^{(n)}}\right) \leqq P\left(D_{i}^{\prime}\right) \sum_{k \geqq n} \mu\left(\mathcal{O}_{m_{k}^{(n)}}\right) \\
& \leqq P\left(D_{i}^{\prime}\right) a_{1}^{(n)}<P\left(D_{i}^{\prime}\right) \varepsilon
\end{aligned}
$$

by Lemma 4.5. This gives the estimate

$$
0 \leqq 1-\frac{P\left(D_{i}^{\prime} \cap \mathscr{2}_{+}^{(n)}\right)}{P\left(D_{i}^{\prime}\right)}<\varepsilon
$$

and hence

$$
0 \leqq \frac{P\left(D_{i}^{\prime}\right)}{P\left(D_{i}^{\prime} \cap \mathscr{Q}_{+}^{(n)}\right)}-1<\frac{\varepsilon}{1-\varepsilon}
$$

Similarly, for any $A \in \mathfrak{B}_{\left[-n^{\prime},-n_{1}\right)}$,

$$
0 \leqq \frac{P\left(A \cap D_{i}^{\prime}\right)}{P\left(D_{i}^{\prime}\right)}-\frac{P\left(A \cap D_{i}^{\prime} \cap \mathscr{Q}_{+}^{(n)}\right)}{P\left(D_{i}^{\prime}\right)}<\varepsilon
$$

and hence,

$$
\left|P\left(A / D_{i}^{\prime}\right)-P\left(A / D_{i}^{\prime} \cap \mathscr{Q}_{+}^{(n)}\right)\right|<\varepsilon+\frac{\varepsilon}{1-\varepsilon},
$$

i.e.,

$$
\operatorname{Var}\left[P^{\left[-n^{\prime},-n_{1}\right)}\left(\cdot / D_{i}^{\prime}\right), P\left(\cdot / D_{i}^{\prime} \cap \mathscr{Q}_{+}^{(n)}\right)\right]<\varepsilon+\frac{\varepsilon}{1-\varepsilon} .
$$

Here $P\left(A / D_{i}^{\prime}\right)$ (resp., $\left.P\left(A / D_{i}^{\prime} \cap \mathscr{Q}_{+}^{(n)}\right)\right)$ and $P^{\left[-n^{\prime},-n_{1}\right)}\left(\cdot / D_{i}^{\prime}\right)$ (resp., $P^{\left[-n^{\prime},-n_{1}\right)}\left(\cdot / D_{i}^{\prime} \cap\right.$ $\left.\mathscr{Q}_{+}^{(n)}\right)$ ) denote the conditional probability of $A$ under $D_{i}^{\prime}$ (resp., $D_{i}^{\prime} \cap \mathcal{Q}_{+}^{(n)}$ ) and the conditional distribution on $\mathfrak{B}_{\left[-n^{\prime},-n_{1}\right)}$ under $D_{i}^{\prime}$ (resp., $D_{i}^{\prime} \cap \mathscr{Q}_{+}^{(n)}$ ) respectively.

Since

$$
\begin{aligned}
& \operatorname{Var}\left[P^{\left[-n^{\prime},-n_{1}\right)}\left(\cdot / D_{i}^{\prime} \cap \mathscr{Q}_{+}^{(n)}\right), P^{\left[-n^{\prime},-n_{1}\right)}\right] \\
& \leqq P\left(D_{i}^{\prime} \cap \mathscr{Q}_{+}^{(n)}\right)^{-1} \int_{D_{i}^{\prime} \cap \mathscr{Q}_{+}^{(n)}} P(d X) \operatorname{Var}\left[\mathscr{P}_{(-\infty, 0)}^{\left[-n^{\prime},-n_{1}\right)}(A ; X), P^{\left[-n^{\prime},-n_{1}\right)}\right],
\end{aligned}
$$

we obtain, by Lemma 4.5 and the above estimate,

$$
\operatorname{Var}\left[P^{\left[-n^{\prime},-n_{1}\right)}\left(\cdot / D_{i}^{\prime}\right), P^{\left[-n^{\prime},-n_{1}\right)}\right]<2 \varepsilon+\frac{\varepsilon}{1-\varepsilon} .
$$

Finally, for $D_{i}^{\prime} \pitchfork E^{(n)}$

$$
\sum_{j}\left|P\left(D_{j}^{\prime \prime} / D_{i}^{\prime}\right)-P\left(D_{j}^{\prime \prime}\right)\right|<2 \operatorname{Var}\left[P^{\left[-n^{\prime},-n_{1}\right)}\left(\cdot / D_{i}^{\prime}\right), P^{\left[-n^{\prime},-n_{1}\right)}\right],
$$

and hence, $\eta_{l}$ is weak Bernoulli. 
Acknowledgements. The author would like to thank Prof. R. Stora for the hospitality at Centre de Physique Théorique of C.N.R.S. in Marseille, and Prof. A. Grossmann for the help with English.

\section{References}

1. Dobrushin, R.L.: The description of a random field by means of conditional probabilities and conditions of its regularity. Theory Probability Appl. 13, 197-224 (1968)

2. Dobrushin, R.L.: Prescribing a system of random variables by conditional distributions. Theory Probability Appl. 15, 458-486 (1970)

3. Dobrushin, R.L.: Gibbsian random fields for lattice systems with mutual interaction. Funct. Anal. Appl. 2, 31-43 (1968)

4. Dobrushin, R.L., Uniqueness of Gibbsian field and a problem of phase transitions. Funct. Anal. Appl. 2, 44-57 (1968)

5. Dobrushin, R.L.: Gibbsian fields: the general case. Funct. Anal. Appl. 3, 27 -35 (1969)

6. Dobrushin, R.L.: Conditions of the absence of phase transitions in one-dimensional classical systems. Math. Sbornik (Russian) 93, 29-49 (1974)

7. Lanford,O.E., Ruelle,D.: Observables at infinity and states with short range correlations in statistical mechanics. Commun. math. Phys. 13, 194-215 (1969)

8. Ruelle, D.: Superstable interactions in classical statistical mechanics. Commun. math. Phys. 18, 127-159 (1970)

9. Averintsev, M. B.: Description of Markovian random fields by Gibbsian conditional probabilities. Theory Probability Appl. 17, 20-33 (1972)

10. Sullivan, W.G.: Potentials for almost Markovian random fields. Commun. math. Phys. 33, 61-74 (1973)

11. Kozlov, O.K.: Gibbs description of random point fields. Theory Probability Appl. (Russian) 21, $348-365$ (1976)

12. Dyson, F.: Existence of a phase-transition in a one-dimensional Ising ferromagnet. Commun. math. Phys. 12, 91-107 (1969)

13. Fisher, M.E., Felderhof, B. U.: Phase transition in one-dimensional cluster-interacting fluids, IA, IB, II, III. Ann. Phys. 58, 176-300 (1970)

14. Gallavotti, G., Miracle-Sole, S.: Absence of phase transitions in hard core one-dimensional systems with long-range interactions. J. Math. Phys. 11, 147-154 (1969)

15. Suhov, Yu. M.: Matrix method for continuous systems of statistical mechanics. Trans. Moscow Math. Soc. (Russian) 24, 175-200 (1971)

16. Ruelle,D.: Statistical mechanics of a one-dimensional lattice gas. Commun. math. Phys. 9, 267-278 (1968)

17. Kerstan,J., Matthes, K., Möcke,J.: Unbegrenzt teilbare Punktprozesse. Berlin: AcademieVerlag 1974

18. Lanford, O.E.: The classical mechanics of one-dimensional systems of infinitely many particles, II. Kinetic theory. Commun. math. Phys. 11, 257-292 (1969)

19. Gikhman,I.I., Skorokhod, A.V.: Theory of stochastic processes, Vol. I (Russian). Moscow: "Nauka" Edition 1971

20. Ornstein, D.: Ergodic theory, randomness, and dynamical systems. New Haven and London: Yale University Press 1974

21. Krein, M.G., Rutman,M.A.: Linear operators leaving invariant a cone in a Banach space. In: Functional Analysis and Measure Theory, AMS Transl. ser. 1, vol. 10, pp. 199-325. Providence, R.I. : 1962

22. Suhov, Yu. M.: Limit density matrices for one-dimensional continuous systems of quantum statistical mechanics. Math. Sbornik (Russian) 83, 491--512 (1970)

23. Caldiera, G., Presutti,E.: Gibbs processes and generalized Bernoulli flows for hard core onedimensional systems. Commun. math. Phys. 35, 279-286 (1974)

24. Ornstein,D.: Two Bernoulli shifts with infinite entropy are isomorphic. Adv. Math. 5, 339-348 (1971)

Communicated by G. Gallavotti

Received February 27, 1976 